\title{
Low-Reynolds-number flow past cylindrical bodies of arbitrary cross-sectional shape
}

\author{
By S. H. LEE† AND L. G. LEAL \\ Chemical Engineering Department, California Institute of Technology, \\ Pasadena, CA 91125, USA
}

(Received 1 May 1985 and in revised form 8 October 1985)

A numerical implementation of the method of matched asymptotic expansions is proposed to analyse two-dimensional uniform streaming flow at low Reynolds number past a straight cylinder (or cylinders) of arbitrary cross-sectional shape. General solutions for both the Stokes and Oseen equations in two dimensions are expressed in terms of a boundary distribution of fundamental single- and double-layer singularities. These general solutions are then converted to integral equations for the unknown distributions of singularity strengths by application of boundary conditions at the cylinder surface, and matching conditions between the Stokes and Oseen solutions. By solving these integral equations, using collocation methods familiar from three-dimensional application of 'boundary integral' methods for solutions of Stokes equation, we generate a uniformly valid approximation to the solution for the whole domain.

We demonstrate the method by considering, as numerical examples, uniform flow past an elliptic cylinder, uniform flow past a cylinder of rectangular cross-section, and uniform flow past two parallel cylinders which may be either equal in radius, or of different sizes.

\section{Introduction}

For creeping flows, the Stokes' equation can be solved exactly using expansions of harmonic functions (Lamb 1932). However, if the boundary does not consist of simple geometric shapes, the exact solution in harmonic functions becomes extremely difficult (or impossible) to implement. Even such relatively simple geometries as two spheres or two circular cylinders (cf. Jeffery 1912; O'Neill 1964; Umemura 1982) are not easy to treat using eigenfunction expansions. There is, however, an alternative approach for the solution of such problems, based upon the use of boundary distributions (or in some cases 'internal' distributions) of fundamental singularities (cf. Oseen 1927; Finn 1959; Chang \& Finn 1961; Ladyzhenskaya 1963). When the boundary shapes are simple enough, this method can be implemented analytically. In more general circumstances, numerical analysis is required to determine the specific distribution of singularities that is necessary to satisfy boundary conditions, but in this so-called 'boundary integral' mode the method can be used, in principle, for boundaries of arbitrary shape.

Indeed, in the case of flows in three-dimensional or axisymmetrical domains, the 'boundary integral' method has already been used to solve a number of difficult, creeping-flow problems using the general integral form of the solution to Stokes equations. In Ladyzhenskaya's (1963) formulation, the velocity field for an arbitrary

† Permanent address: Chevron Oil Field Research Co., PO Box 446, La Habra, CA 90631. 
three-dimensional creeping motion can be determined completely by evaluating the distribution of so-called single- and double-layer singularities over the boundaries of the flow domain. One advantage of this particular formulation is that the intensities of the single- and double-layer potentials correspond directly to the velocity and stress components at the boundaries, and thus hydrodynamic forces or torques are evaluated easily once the single- and double-layer intensities have been determined. Ladyzhenskaya's general solution has been applied to a large variety of interesting creeping-flow problems: for example the slow viscous flow of an unbounded fluid past a single solid body by Youngren \& Acrivos (1975), the motion of a sphere in the presence of a deformable interface by Lee \& Leal (1982), and the deformation of a liquid drop in shear flows by Rallison (1981).

For two-dimensional flows, on the other hand, it is well known that the solution of Stokes' equation cannot simultaneously satisfy the no-slip conditions at a body surface and still yield a bounded uniform velocity field at infinity. In other words, Stokes' equation does not provide a uniformly valid first approximation for the velocity field in the whole domain as is true for three-dimensional problems. Owing to Stokes' paradox, an effective 'boundary-integral' method has not yet been developed for two-dimensional flows in an unbounded domain. Of course, an effective remedy to Stokes' paradox has been known for many years in the context of analytical studies using the method of singular perturbation (or matched asymptotic) expansions. This method resolves the problem with boundary conditions at infinity by using the Oseen approximation for the outer field, thus restricting Stokes' approximation to an inner field within a distance $O\left(l_{\text {body }}\right)$ from the body surface (cf. Proudman \& Pearson 1957 ; Kaplun 1957). Using this method, analytic solutions have been obtained for many two-dimensional flows past bodies of relatively simple geometry where the body surface is coincident with a coordinate surface. For example, solutions have been obtained for both streaming (uniform) flow and simple shear flow past a circular cylinder (Robertson \& Acrivos 1970), and, more recently, for streaming flow past two equal circular cylinders using bipolar cylindrical coordinates (Umemura 1982).

In the present paper, we show how a numerical implementation of the method of matched asymptotic expansions can be used to consider uniform streaming flow at low Reynolds number past straight cylinders of arbitrary cross-sectional geometry. General solutions of Stokes' and Oseen's equations for two-dimensional flows are first obtained in terms of distributions of single- and double-layer singularities at the boundaries. Then, by using the asymptotic matching principle between Stokes' solution for the inner field and Oseen's solution for the outer field, we obtain a uniformly valid solution for the whole domain. The method allows all the flow field to be determined completely at each level of approximation in both the inner and outer domains by evaluating distributions of singularities over the cylinder surface. The no-slip boundary condition is included easily in solving for the strengths of singularities at the boundaries. Furthermore, by systematic procedure, we can calculate not only the leading-order solutions, but also higher-order solutions for low-Reynolds-number flows.

Even though two-dimensional flow problems can be solved using a stream-function formulation, we use the velocity components and pressure as the primary variables. This 'direct velocity method' yields a larger matrix to invert in calculating the boundary distributions for the single- and double-layer potentials. However, in order to evaluate stresses, a solution in terms of the stream function would require more numerical differentiation which may be expected to cost a great deal in terms of accuracy. 


\section{Formulation}

We consider then a uniform flow past a cylinder(s) of arbitrary shape in the limit of arbitrarily small Reynolds numbers. Without losing generality, we assume that the uniform velocity at infinity is in the $x_{1}$-coordinate direction.

$$
u^{\prime}=U_{\infty} e_{1} \text { at } x \rightarrow \infty .
$$

The steady Navier-Stokes equation in dimensionless form is

$$
0=-\nabla p+\nabla^{2} u-R e(u \cdot \nabla u)
$$

and the equation of continuity is

$$
0=\boldsymbol{\nabla} \cdot \boldsymbol{u} .
$$

Equations (2) and (3) are non-dimensionalized with respect to characteristic variables $u_{\mathrm{c}}=U_{\infty}, l_{\mathrm{c}}=a$ (characteristic cylinder radius), and $p_{\mathrm{c}}=\mu U_{\infty} / a$. The Reynolds number is defined as

$$
R e \equiv \frac{U_{\infty} a}{\nu} .
$$

In the inner region, since the inertia term is small (i.e. $R e \ll 1$ ), we can assume an expansion of the form

$$
(u, p)=f_{0}(R e)(u, p)^{0}+f_{1}(R e)(u, p)^{1}+\ldots,
$$

where the leading-order term satisfies Stokes' equation,

$$
\begin{aligned}
& 0=-\nabla p^{0}+\nabla^{2} u^{0}, \\
& 0=\nabla \cdot u^{0},
\end{aligned}
$$

and the governing equation for the next term $(u, p)^{1}$ is expressed readily as

$$
\begin{aligned}
& 0=-\nabla p^{1}+\nabla^{2} u^{1}-R e \frac{f_{0}(R e)^{2}}{f_{1}(R e)} u^{0} \cdot \nabla u^{0}, \\
& 0=\nabla \cdot u^{1} .
\end{aligned}
$$

Equation (7) for $(u, p)^{1}$ is simply Stokes' equation with an inhomogeneous term $\operatorname{Re}\left(f_{0}^{2} / f_{1}\right) \boldsymbol{u}^{0} \cdot \nabla u^{0}$.

In the outer region, $|x| \geqslant O\left(R e^{-1}\right)$, the expansion (4) is not valid, as is well known, because the inertia term in (2) becomes as important as the viscous term. In this region we choose characteristic variables $\hat{l}_{\mathrm{c}}=a / R e, \hat{p}_{\mathrm{c}}=\mu U_{\infty} / t_{\mathrm{c}}=\operatorname{Re} p_{\mathrm{c}}$, and $\hat{u}_{\mathrm{c}}=U_{\infty}$, and the governing equations become

$$
\begin{aligned}
& 0=\hat{\nabla} \hat{p}+\hat{\nabla}^{2} \hat{a}-\hat{a} \cdot \hat{\nabla} \hat{u}, \\
& 0=\hat{\nabla} \cdot \hat{a} .
\end{aligned}
$$

Here, the gradient operator is defined in terms of spatial variables scaled with respect to $\boldsymbol{l}$, i.e. $\hat{\boldsymbol{v}} \equiv \partial / \partial \hat{\boldsymbol{x}}$. We seek a solution for $(\hat{\boldsymbol{u}}, \hat{\boldsymbol{p}})$ in the form of an asymptotic expansion

where

$$
\begin{aligned}
(\hat{u}, \hat{p})= & \hat{f}_{0}(R e)(\hat{u}, \hat{p})^{0}+\hat{f}_{1}(R e)(\hat{u}, \hat{p})^{1}+\ldots, \\
& \frac{\hat{f}_{n+1}(R e)}{\hat{f}_{n}(R e)} \rightarrow 0, \quad \text { as } R e \rightarrow 0 .
\end{aligned}
$$

The obvious choice for the first term in (11) is the uniform flow, i.e.

$$
\begin{aligned}
& \hat{f}_{0}(R e)=1, \\
& (\hat{u}, \hat{p})^{0}=\left(e_{1}, 0\right) .
\end{aligned}
$$


Substituting the expansion (11) into (9) and (10), using (12) and (13), we obtain Oseen's equation for the second-order approximation,

$$
\begin{aligned}
& 0=-\hat{\nabla} \hat{p}^{1}+\hat{\nabla}^{2} \hat{u}^{1}-\frac{\partial \hat{u}^{1}}{\partial \hat{x}_{1}}, \\
& 0=\hat{\nabla} \cdot \hat{\boldsymbol{u}}^{1} .
\end{aligned}
$$

Although Stokes' equation is a valid approximation only for the inner field whereas Oseen's equation applies for the outer field, the familiar asymptotic matching procedure yields a uniform approximation which is valid for the whole domain.

In the following sections, we will first discuss general solutions of Stokes' and Oseen's equations in the boundary-integral form for two-dimensional flows. Then, we show how to apply these solutions in a numerical implementation of the method of matched asymptotic expansions to obtain solutions for streaming flow past cylindrical bodies of arbitrary cross-sectional geometry.

\section{A general solution of Stokes' equation in two dimensions}

We have seen that the leading-order approximation to the velocity and pressure in the inner domain satisfies the Stokes' equation which completely neglects the inertia term

$$
\begin{aligned}
& 0=-\boldsymbol{\nabla} p+\nabla^{2} \boldsymbol{u}, \\
& 0=\boldsymbol{\nabla} \cdot \boldsymbol{u} .
\end{aligned}
$$

For three-dimensional flow, a fundamental solution corresponding to a point force in (16) can be derived easily using Fourier transforms (cf. Ladyzhenskaya 1963).

The corresponding fundamental solution of Stokes' equation for a two-dimensional flow can be derived in a similar manner, and we shall not dwell on the details here. If we consider a point force in the $e_{k}$-direction applied at $x=y$, i.e. $-\delta(x-y) e_{k}$, then the fundamental solution in two dimensions is

$$
\begin{aligned}
v_{i}^{k} & =\frac{1}{4 \pi}\left[\log |x-y| \delta_{i}^{k}-\frac{\left(x_{i}-y_{i}\right)\left(x_{k}-y_{k}\right)}{|x-y|^{2}}\right], \\
q^{k} & =-\frac{1}{2 \pi} \frac{x_{k}-y_{k}}{|x-y|^{2}} .
\end{aligned}
$$

Here, $v_{i}^{k}$ is the $i$ th component of velocity due to a point force in the $e_{k}$ direction, while $q^{k}$ is the corresponding pressure. It may be noted that $v_{i}^{k}$ has a logarithmic singularity at infinity. If the fundamental solutions, (18) and (19), are used to calculate the stresses in the fluid, we obtain

$$
T_{i j}^{k}=\frac{1}{\pi} \frac{\left(x_{i}-y_{i}\right)\left(x_{j}-y_{j}\right)\left(x_{k}-y_{k}\right)}{|x-y|^{4}}
$$

Again, to be sure that the nomenclature is firmly in mind, we note that $T_{i j}^{k}$ is the $i j$ component of the stress due to the motion induced by a point force at $x=y$ that is applied in the $e_{k}$-direction.

Now, using Green's theorem, we can obtain a general solution of Stokes' equation 
for two-dimensional flows in terms of hydrodynamic potentials which derive from distributions of singularities at the boundaries,

$$
\begin{array}{r}
u(x)=\frac{1}{4 \pi} \int_{\Gamma}\left[\left(\log \frac{1}{r}\right) I+\frac{(x-\eta)(x-\eta)}{r^{2}}\right] \cdot T(\eta) \cdot n \mathrm{~d} \Gamma_{\eta} \\
-\frac{1}{\pi} \int_{\Gamma} \frac{(x-\eta)(x-\eta)(x-\eta)}{r^{4}} \cdot u(\eta) \cdot n \mathrm{~d} \Gamma_{\eta}, \\
p(x)=\frac{1}{2 \pi} \int_{\Gamma} \frac{(x-\eta)}{r^{2}} \cdot T(\eta) \cdot n \mathrm{~d} \Gamma_{\eta}+\frac{1}{\pi} \int_{\Gamma}\left(\frac{I}{r^{2}}-\frac{2(x-\eta)(x-\eta)}{r^{4}}\right) \cdot u(\eta) \cdot n \mathrm{~d} \Gamma_{\eta} .
\end{array}
$$

Here, $\Gamma$ represents the boundaries of the flow domain and $r$ is defined as $|x-\eta|$. The unit normal $n$ is an outer normal to the flow domain at $\Gamma$.

These general solutions of Stokes' equation, (21) and (22), are expressed in terms similar to Fredholm integrals of the second kind. The first integral in (21) and (22) is denoted as the single-layer contribution, whereas the second integral is known as the double-layer contribution.

For three-dimensional flow, Ladyzhenskaya (1965) noted that the single-layer potential was continuous across a flow boundary, while the double-layer potential experienced a discontinuous jump at the boundary. If we define $W(x)$ as

$$
W(x)=-\frac{1}{\pi} \int_{\Gamma} \frac{(x-\eta)(x-\eta)(x-\eta)}{r^{4}} \cdot u \cdot n \mathrm{~d} \Gamma_{n},
$$

for two-dimensional flow, the jump condition for the double-layer potential is given by

$$
\begin{aligned}
& W_{i}(\zeta)=\frac{1}{2} u(\zeta)+W_{s}(\zeta), \\
& W_{e}(\zeta)=-\frac{1}{2} u(\zeta)+W_{s}(\zeta)
\end{aligned}
$$

Here

$$
\begin{array}{ll}
W_{i}(\zeta) \equiv \lim _{x \rightarrow \zeta} W(x) & \text { for } x \in \Omega \\
W_{e}(\zeta) \equiv \lim _{x \rightarrow \zeta} W(x) & \text { for } x \notin \bar{\Omega}
\end{array}
$$

and $W_{s}(\zeta)$ is $W(\zeta)$ evaluated at $x=\zeta \in \Gamma$.

We note that the intensities of the single and double layers are, respectively, the stress and velocity components at the boundary. Equation (21) is of lower order in spatial dimension than the full creeping-motion equation. If we apply (21) at the boundaries where some components of $\boldsymbol{u}$ and/or $\boldsymbol{T}$ can be specified from the boundary conditions, the unknown components of $u$ and $T$ at the same boundaries can then be calculated directly by solving the resulting integral equations. These boundary values of $u$ and $T$ are frequently the desired information; for example, we may wish to evaluate the hydrodynamic drag and torque on a body. Once we obtain the strengths of the singularities at the boundaries, the velocity or pressure distributions in the interior of the domain can be calculated easily from the integral expressions (21) and (22).

For three-dimensional flows, the corresponding integral solution due to Ladyzhenskaya has been used successfully to solve a variety of creeping-flow problems (cf. Youngren \& Acrivos 1975 ; Rallison 1981 ; Lee \& Leal 1982). Nevertheless, so far as we are aware, there has been no similar application of (21) and (22) owing to the fact that the Stokes' approximation is not valid for the flow field far away from the body 
in an unbounded fluid. It is evident from (21) that the general solution of Stokes' equation for two-dimensional flows involves a logarithmic singularity at infinity. For a bounded flow field, of course, a solution can be obtained from (21), without reference to a singular perturbation. However, owing to the logarithmic term, the linear matrix into which a collocation method transforms (21) will not be diagonally dominant as for three-dimensional flows. Hence, the Stokes' solution for two-dimensional flow will require careful numerical evaluation to minimize numerical error.

\section{A general solution of Oseen's equation for two-dimensional flow}

The inertial term becomes as important as the viscous term in the equation of motion in the flow field far away from a body, i.e. $|x| \geqslant O\left(R e^{-1}\right)$. Oseen (1927) proposed the so-called Oseen's equation to approximate the leading-order inertial term for low-Reynolds-number flows,

$$
\begin{aligned}
& 0=-\nabla \hat{p}+\nabla^{2} \hat{u}-\frac{\partial \hat{u}}{\partial \hat{x}_{1}}, \\
& 0=\nabla \cdot \hat{u},
\end{aligned}
$$

and we have seen that this equation governs the leading-order departure from the free-stream velocity in the outer region of a matched asymptotic expansion scheme. Although the Oseen's equation is a correct approximation only for the flow field far from a body, Proudman \& Pearson (1957) showed that the drag calculated on the basis of Oseen's equation with no-slip boundary conditions applied at the body surface still yields a correct higher-order approximation for the force on a solid sphere in a uniform flow. Yano \& Kieda (1980) numerically solved Oseen'sequation by distributing Oseenlet singularities inside a two-dimensional body and approximately applying the no-slip boundary conditions at the solid surface using a least-squares regression. The derivation in this section can certainly be adopted to evaluate a flow field generated solely on the basis of Oseen's equation. However, in the present paper, we are concerned with the solution of Oseen's equation in the context of a singular perturbation (matched asymptotic expansion) scheme in which Oseen's equation applies in the outer region and is matched with the corresponding Stokes' solution for $\hat{r}=r R e=O(1)$.

A fundamental solution of Oseen's equation for two-dimensional flow can be derived formally by including a point force $-e_{k} \delta(\hat{x}-\hat{y})$, on the right-hand side of (26) and then using Fourier transforms. The result is

$$
\begin{gathered}
\hat{v}_{i}^{k}=-\frac{1}{4 \pi} \mathrm{e}^{\frac{1}{2}\left(\hat{x}_{1}-\hat{y}_{1}\right)} K_{0}\left(\frac{1}{2} \rho\right) \delta_{i}^{k} \\
-\frac{1}{4 \pi}\left(\mathrm{e}^{\frac{1}{2}\left(\hat{x}_{1}-\hat{y}_{1}\right)} K_{1}\left(\frac{1}{2} \rho\right)-\frac{2}{\rho}\right)\left\{\frac{\hat{x}_{i}-\hat{y}_{i}}{\rho} \delta_{k}^{i}+\frac{\left(\hat{x}_{2}-\hat{y}_{2}\right) \delta_{i}^{1}-\left(\hat{x}_{1}-\hat{y}_{1}\right) \delta_{i}^{2}}{\rho} \delta_{k}^{2}\right\}, \\
\hat{q}^{k}=-\frac{1}{2 \pi} \frac{\left(\hat{x}_{k}-\hat{y}_{k}\right)}{\rho^{2}},
\end{gathered}
$$

where $\rho=|\hat{x}-\hat{y}| . K_{0}$ and $K_{1}$ are modified Bessel functions of the second kind, of order 0 and 1 , respectively. The adjoint of (26) and (27) including a point force can be written as

$$
\begin{aligned}
& 0=\nabla \hat{p}^{*}+\nabla^{2} \hat{u}^{*}+\frac{\partial \hat{u}^{*}}{\partial \hat{x}_{1}}-e_{k} \delta(\hat{x}-\hat{y}), \\
& 0=\nabla \cdot \hat{u}^{*}
\end{aligned}
$$


The solution of these adjoint equations (30) and (31) can be derived similarly:

$$
\begin{aligned}
\hat{u}_{i}^{k *}=-\frac{1}{4 \pi} \mathrm{e}^{-\frac{1}{2}\left(\hat{x}_{1}-\hat{y}_{1}\right)} K_{0}\left(\frac{1}{2} \rho\right) \delta_{i}^{k} & \\
+\frac{1}{4 \pi}\left(\mathrm{e}^{-\frac{1}{2}\left(\hat{x}_{1}-\hat{y}_{1}\right)} K_{1}\left(\frac{1}{2} \rho\right)-\right. & \frac{2}{\rho}\left\{\frac{\left(\hat{x}_{i}-\hat{y}_{i}\right)}{\rho} \delta_{k}^{1}+\frac{\left(\hat{x}_{2}-\hat{y}_{2}\right) \delta_{i}^{1}-\left(\hat{x}_{1}-\hat{y}_{1}\right) \delta_{i}^{2}}{\rho} \delta_{k}^{2}\right\}, \\
\hat{p}^{k *} & =\frac{1}{2 \pi} \frac{\left(\hat{x}_{k}-\hat{y}_{k}\right)}{\rho^{2}} .
\end{aligned}
$$

In addition, the stress from the adjoint system becomes

Then,

$$
\hat{T}_{i j}^{*}\left(\hat{u}^{k *}\right) \equiv \hat{p}^{k * \delta_{i}^{\gamma}}+\left(\frac{\partial \hat{u}_{i}^{k *}}{\partial \hat{x}_{j}}+\frac{\partial \hat{u}_{j}^{k *}}{\partial \hat{x}_{i}}\right)
$$

$$
\begin{aligned}
\hat{T}_{i j}^{*}\left(\hat{u}^{1 *}\right)=\frac{1}{2 \pi} & \frac{\hat{d}_{1}}{\rho^{2}} \delta_{i}^{j}-\frac{1}{\pi \rho^{2}}\left(\delta_{i}^{j}-\frac{2 \hat{d}_{i} \hat{d}_{j}}{\rho^{2}}\right) \\
& +\frac{1}{4 \pi} \mathrm{e}^{-\frac{1}{2} \hat{d}_{1}} K_{0}\left(\frac{1}{2} \rho\right)\left(\delta_{i}^{1} \delta_{j}^{1}-\frac{\hat{d}_{i} \hat{d}_{j}}{\rho^{2}}\right)+\frac{1}{2 \pi} \mathrm{e}^{-\frac{1}{2} d_{1}} K_{1}\left(\frac{1}{2} \rho\right)\left(\frac{\delta_{i}^{y}}{\rho}-\frac{2 \hat{d}_{i} \hat{d}_{j}}{\rho^{3}}\right),
\end{aligned}
$$

and

$$
\begin{aligned}
\hat{T}_{i j}^{*}\left(\hat{u}^{2 *}\right)= & \frac{1}{2 \pi} \frac{\hat{d}_{2}}{\rho^{2}} \delta_{i}^{j}-\frac{1}{\pi \rho^{4}}\left\{\hat{d}_{1}\left(\delta_{i}^{2} \hat{d}_{j}+\delta_{j}^{2} \hat{d}_{i}\right)-\hat{d}_{2}\left(\delta_{i}^{1} \hat{d}_{j}+\delta_{j}^{1} \hat{d}_{i}\right)\right\}\left\{1-\frac{1}{8} \rho^{2} \mathrm{e}^{-\frac{1}{2} \hat{d}_{1}} K_{0}\left(\frac{1}{2} \rho\right)\right\} \\
& +\frac{1}{8 \pi} \mathrm{e}^{-\frac{1}{2} a_{1}} K_{0}\left(\frac{1}{2} \rho\right)\left(\delta_{i}^{1} \delta_{j}^{2}+\delta_{i}^{2} \delta_{j}^{1}\right)+\frac{1}{8 \pi} \frac{\mathrm{e}^{-\frac{1}{2}} \hat{d}_{1}}{\rho} K_{1}\left(\frac{1}{2} \rho\right)\left\{\delta_{i}^{2} \hat{d}_{j}+\delta_{j}^{2} \hat{d}_{i}\right. \\
& \left.+\left(\delta_{i}^{1}-\frac{4 \hat{d}_{i}}{\rho^{2}}\right)\left(\hat{d}_{2} \delta_{j}^{1}-\hat{d}_{1} \delta_{j}^{2}\right)+\left(\delta_{j}^{1}-\frac{4 \hat{d}_{j}}{\rho^{2}}\right)\left(\hat{d}_{2} \delta_{i}^{1}-\hat{d}_{1} \delta_{i}^{2}\right)\right\} .
\end{aligned}
$$

Here, $\hat{d}_{i} \equiv \hat{x}_{i}-\hat{y}_{i}$. Using Green's theorem and (32)-(36), a general solution of Oseen's equation can now be derived,

$$
\hat{u}_{k}(\hat{x})=-\int_{\hat{\Gamma}} \hat{T}(\hat{u}) \cdot \hat{u}^{k *} \cdot n \mathrm{~d} \hat{\Gamma}-\int_{\hat{\Gamma}} \hat{T}^{*}\left(\hat{u}^{k *}\right) \cdot \hat{u} \cdot n \mathrm{~d} \hat{\Gamma}+\int_{\hat{\Gamma}}\left(\hat{u} \cdot \hat{u}^{k *}\right) n_{1} \mathrm{~d} \hat{\Gamma} .
$$

Here, $\hat{\Gamma}$ is the boundary of flow domain in the outer variables. By substituting (32), (35) and (36) into (37), we can readily solve for $\hat{u}$ or $\hat{T}(\hat{u})$ on $\hat{\Gamma}$. From (29) and (33), a general expression for the pressure can be derived as

$$
\begin{aligned}
& \hat{p}(\hat{x})=\int_{\tilde{\Gamma}}\left(2 \nabla_{x} \hat{p}^{*}+\hat{p}^{1 * I}\right) \cdot \hat{u} \cdot n \hat{\Gamma}-\int_{\hat{\Gamma}} \hat{p}^{* \cdot \hat{T}} \cdot n \hat{\Gamma}+\int_{\hat{\Gamma}} \hat{p}^{*} \cdot \hat{u} n_{1} \hat{\Gamma} \\
& \text { for } \hat{p}^{*} \equiv\left(\hat{p}^{1 *}, \hat{p}^{2 *}\right) \text {. }
\end{aligned}
$$

The first and second terms on the right-hand side of (37) correspond, respectively, to the single-layer and double-layer terms of Stokes' solution in (21). It can also be proved that the single-layer contribution to Oseen's solution is continuous across the boundary, while the double-layer contribution has a discontinuous jump. Since a uniform velocity field $\hat{u}=\boldsymbol{c}$ is a solution of (37), the jump condition for the double-layer term is clearly identical to (24) and (25).

If we were interested in solving Oseen's equation with no-slip boundary conditions applied on a cylindrical boundary $(\hat{\Gamma})$, as in Yano \& Kieda (1980), the integral equation resulting from the application of boundary conditions in (37) could be solved 
easily by a collocation method. However, in the present work, we are concerned with the solution of Oseen's equation in the context of a matched asymptotic solution in which Oseen's solution is utilized only in the outer portion of the fluid domain.

\section{Application of the fundamental solutions to flow past an arbitrary two-dimensional body via the method of matched asymptotic expansions}

Proudman \& Pearson (1957) and Kaplun (1957) devised the method of matched asymptotic expansions to obtain higher-order approximations to the solution for low-Reynolds-number flow past a sphere or a circular cylinder. In this section, we utilize the same singular-perturbation method in order to evaluate the higher-order terms in (4) and (11) for flows past an arbitrary two-dimensional body. Although the method is, in principle, capable of systematically calculating terms of any order in $R e$, the present paper will carry the approximation only up to the second term in $(4), f_{1}(R e)(u, p)^{1}$, which is a first correction to the Stokes' solution.

As discussed in $\$ 2$, the zeroth-order solution for the outer region is a uniform flow,

$$
\begin{aligned}
\hat{f}_{0}(R e) & =1, \\
\left(\hat{\boldsymbol{u}}^{0}, \hat{p}^{0}\right) & =\left(\boldsymbol{e}_{1}, 0\right) .
\end{aligned}
$$

Thus, matching requires that the zeroth-order solution for the inner region should become

$$
\lim _{|x| \gg 1} f_{0}(R e)\left(u^{0}, p^{0}\right) \leftrightarrow\left(e_{1}, 0\right), \quad \text { as } R e \rightarrow 0 .
$$

Let us now consider how to obtain a solution of Stokes' equation for $u^{0}$ and $p^{0}$ which satisfies the matching condition (41), as well as the no-slip condition on a cylindrical body of arbitrary cross-sectional shape. To do this, we first digress and consider the form of the Stokes' velocity field that is generated, in two dimensions, by the translation of an arbitrary cylindrical body with unit velocity in the orthogonal 1 and 2 directions (i.e. in the plane orthogonal to the axis of the body, with the 1 direction coincident with the direction of uniform motion in the original problem). In other words, we consider the solutions of Stokes' equation which satisfies the condition

$$
\tilde{u}_{(j)}=-e_{j}, \quad \text { on } x \in \Gamma
$$

for $j=1$ and 2 . We shall call these velocity fields the 'disturbance' velocity fields for reasons that will soon become apparent. Since there do not exist any singularities except on $\Gamma$, the general form for $\tilde{u}_{(j)}$ can be seen from (21) to be

$$
\tilde{u}_{(j)}=\frac{1}{4 \pi} \int_{\Gamma}\left[\left(\log \frac{1}{r}\right) I+\frac{(x-\eta)(x-\eta)}{r^{2}}\right] \cdot \tau_{(j)} \cdot n \mathrm{~d} \Gamma .
$$

The double-layer term which appears in the general solution (21) of Stoke's equations is zero since $\tilde{u}_{(j)}$ is constant on $\Gamma$. The unknown weighting function, $\boldsymbol{T}_{(j)}$ (the 'surface stress') can be determined (for $j=1$ and 2) by solving (43) subject to the boundary condition (42), i.e.

$$
-e_{f}=\frac{1}{4 \pi} \int_{\Gamma}\left[\left(\log \frac{1}{r}\right) I+\frac{(x-\eta)(x-\eta)}{r^{2}}\right] \cdot T_{(j)} \cdot n \mathrm{~d} \Gamma .
$$

We shall show below that a solution of (5) and (6), subject to the matching condition (41) for $\boldsymbol{u}^{0}, p^{0}$, can be constructed using the disturbance-flow velocity fields, $\tilde{\boldsymbol{u}}_{(1)}$ and $\tilde{u}_{(2)}$. To do this it will be useful to have available the asymptotic form of $\tilde{u}_{(j)}$ for large 
$r=O\left(R e^{-1}\right)$ for $R e \rightarrow 0$. To determine this form, we express (43) in terms of outer variables

$$
\tilde{u}_{(j)} \sim \frac{1}{4 \pi} \int_{\Gamma}\left[\left(\log \frac{R e}{\rho}\right) I+\frac{(\hat{x}-\hat{\eta})(\hat{x}-\hat{\eta})}{\rho^{2}}\right] \cdot \hat{T}_{(j)} \cdot n \mathrm{~d} \hat{\Gamma}
$$

Here, $T$ denotes the stress, $T$ expressed in terms of outer variables, i.e.

$$
\hat{T}(\hat{x})=\frac{1}{R e} T(x)
$$

It can be noted also that

$$
\begin{gathered}
\mathrm{d} \hat{\Gamma}=\operatorname{Re} \mathrm{d} \Gamma \\
\int_{\Gamma} \boldsymbol{T} \cdot \mathrm{n} \mathrm{d} \Gamma=\int_{\Gamma} \hat{T} \cdot \boldsymbol{n} \mathrm{d} \hat{\Gamma} .
\end{gathered}
$$

Equation (48) indicates that the integrals along the boundary are identical whether the integrands are expressed in terms of inner variables or outer variables. The asymptotically dominant contribution to $\tilde{u}_{(j)}$ is

$$
\tilde{u}_{(j)} \sim \frac{1}{2 \pi}(\log R e) \int_{\Gamma} T_{(j)} \cdot n \mathrm{~d} \Gamma .
$$

Now, $T_{(j)}$ is just the surface stress associated with the motion of an arbitrary two-dimensional body with unit velocity in the direction $-e_{j}$, and the integral $\int T_{(j)} \cdot n \mathrm{~d} \Gamma$ which appears in (49) is thus the net hydrodynamic force on the body, resulting from the same motion. It is therefore evident that the uniform velocity that is generated in the far field (i.e. (49)) by uniform translation of an arbitrary cylindrical body is not co-linear with the direction of motion of the body except when the hydrodynamic lift on the body is zero.

We now attempt to construct the solution $\boldsymbol{u}^{0}$ of the original Stokes'-flow problem as a linear combination of $\tilde{u}_{(1)}$ and $\tilde{u}_{(2)}$ such that the matching condition (41) and the boundary conditions at the cylinder surface are all satisfied. It is evident, from the considerations of the preceding paragraph, that the matching condition (41) can be satisfied only, in general, by some combination of $\tilde{u}_{(1)}$ and $\tilde{u}_{(2)}$, with the exception being cases in which the symmetry of the body is such that there is no hydrodynamic lift. We therefore propose the form

$$
u^{0}=c\left(e_{1}+\alpha e_{2}\right)+c\left(\tilde{u}_{(1)}+\alpha \tilde{u}_{(2)}\right) .
$$

It can be seen from the condition (42) on $\tilde{u}_{(i)}$ that $\boldsymbol{u}^{0}$ satisfies the no-slip condition at the body surface. Furthermore, it is evident from the asymptotic forms (49) for $\tilde{u}_{(1)}$ and $\tilde{u}_{(2)}$ that the matching condition (41) will be satisfied to leading order for $R e \rightarrow 0$ if

and

$$
f_{0}(R e)=\frac{1}{\log R e}
$$

Expressed in component form, the latter condition requires

$$
\int_{\Gamma}\left(\left(\tilde{T}_{2 n}\right)_{(1)}+\alpha\left(\tilde{T}_{2 n}\right)_{(2)}\right) \mathrm{d} \Gamma=0,
$$

and

$$
\frac{c}{4 \pi}\left[\int_{\Gamma}\left(\left(\tilde{T}_{1 n}\right)_{(1)}+\alpha\left(\tilde{T}_{1 n}\right)_{(2)}\right) \mathrm{d} \Gamma\right]=1
$$


The constant $\alpha$ can be determined from $(53 a)$, and $(53 b)$ then yields the required value for $c$. Both constants will generally depend in some complicated way on the geometry of the body. However, it is clear that the coefficient $\alpha$ will be zero in any case where the symmetry of the problem is such that the lift on the body translating in the $e_{1}$ direction is zero.

The complete leading-order solution of the Stokes problem is thus

$$
\begin{aligned}
f_{0}(R e) u^{0}= & \frac{c}{\log R e}\left(e_{1}+\alpha e_{2}\right) \\
& \quad+\frac{c}{\log R e} \int_{\Gamma}\left[\left(\log \frac{1}{r}\right) I+\frac{(x-\eta)(x-\eta)}{r^{2}}\right] \cdot\left(T_{(1)}+\alpha T_{(2)}\right) \cdot n \mathrm{~d} \Gamma
\end{aligned}
$$

The next leading-order terms in (54) for large $|x|$ must be matched with the second term in the outer expansion (11), namely the solution of Oseen's equation (14) and (15). From the asymptotic expansion (11) and the form (54), it is obvious that

$$
f_{1}(R e) \equiv \frac{c_{1}}{\log R e} .
$$

However, to proceed further, it is necessary to consider the asymptotic form of the Oseen's solution, (37), for small $\rho$, namely

$$
\begin{aligned}
\hat{f}_{1}(R e) \hat{u}_{k}^{1}=\frac{c_{1}}{\log R e}( & \left.-\frac{1}{4 \pi}\right) \int\left[(\log \rho+\gamma-\log 4) \delta_{1}^{k}\right. \\
& \left.-\left\{d_{i} \delta_{k}^{1}+\left(d_{2} \delta_{i}^{1}-d_{2} \delta_{i}^{2}\right) \delta_{k}^{2}\right\} \frac{d_{1}}{\rho^{2}}\right] \cdot \hat{T}_{i j} \cdot n_{j} \mathrm{~d} \hat{\Gamma}+O(\rho \log \rho) .
\end{aligned}
$$

Comparing (54), expressed in terms of outer variables, and the small- $\rho$ form of the outer solution, (56), we see that the matching condition at $O(1 / \log R e)$ requires

$$
c_{1}=c
$$

and

$$
\hat{T}_{i j}=\left(\hat{T}_{i j}\right)_{(1)}+\alpha\left(\hat{T}_{i j}\right)_{(2)}, \quad \text { for } x \in \Gamma \text {. }
$$

Finally, proceeding to the next level in the inner solution, we require that the term $f_{1}(R e) u_{1}$ match with the leading-order mismatch between $f_{0}(R e) u_{0}$ and the first two terms $\hat{f}_{0}(R e) \hat{u}_{0}+\hat{f}_{1}(R e) \hat{u}_{1}$, from the outer region. This mismatch is given by

$$
\begin{aligned}
\Delta & \equiv \hat{f}_{0}(R e) \hat{\boldsymbol{u}}_{0}+\hat{f}_{1}(R e) \hat{\boldsymbol{u}}_{1}-f_{0}(R e) \boldsymbol{u}^{0} \\
& =-\frac{c}{\log R e}\left\{\frac{1}{4 \pi} \int_{\Gamma}\left[(\gamma-\log 4) \Pi \cdot \boldsymbol{T}^{1} \cdot \boldsymbol{n} \mathrm{d} \hat{\Gamma}-\left(e_{1}+\alpha e_{2}\right)+O(\rho \log \rho)\right\} .\right.
\end{aligned}
$$

It is clear from (59) and (53b) that the next term in the Stokes' (inner) expansion should be matched with the uniform flow

$$
\Delta \sim-\frac{1}{\log R e}\left\{(\gamma-\log 4-c) e_{1}-c \alpha e_{2}\right\} .
$$

Further, in view of the fact that this 'mismatch' uniform flow is $O(\log R e)^{-1}$, it is evident that $u_{1}$ will satisfy Stokes equation, the inertial term $\boldsymbol{u}^{\mathbf{0}} \cdot \nabla u^{0}$ in (7) being asymptotically negligible compared to any inverse power of $\log R e$. 
We have seen previously that the general solution (21) of Stokes equation with a boundary-stress distribution

$$
f_{0}(R e) T^{0}=\frac{c}{\log R e}\left(T_{(1)}+\alpha T_{(2)}\right)
$$

at the cylinder surface and $\alpha$ satisfying (53a), generates a uniform flow $e_{1}$ at leading order in the matching region. Thus, in order to obtain a solution of Stokes equations which matches the $e_{1}$ component of (60), we require a surface-stress distribution of the form (61) with strength

$$
-\frac{c}{(\log R e)^{2}}(\gamma-\log 4-c)\left(T_{(1)}+\alpha T_{(2)}\right),
$$

in which $\alpha$ again satisfies (53a). In order to obtain a solution of Stokes' equation which generates a uniform flow, $e_{2}$ in the far field, we require a surface stress distribution on the cylinder surface of the form

where

$$
\begin{gathered}
\frac{c_{2}}{\log R e}\left(\beta T_{(1)}+T_{(2)}\right), \\
c_{2}=\left[\frac{1}{4 \pi} \int\left[\beta\left(\tilde{T}_{2 n}\right)_{(1)}+\left(\tilde{T}_{2 n}\right)_{(2)}\right] \mathrm{d} \Gamma\right]^{-1},
\end{gathered}
$$

and $\beta$ is determined from the condition

$$
\int_{\Gamma}\left[\beta\left(\tilde{T}_{1 n}\right)_{(1)}+\left(\tilde{T}_{1 n}\right)_{(2)}\right] \mathrm{d} \Gamma=0 .
$$

Thus, in order to obtain a solution of Stokes' equation which matches the $e_{2}$ uniform velocity contribution in (60), we use the general solution (21) with a stress distribution

and $\beta$ satisfying (65).

$$
\frac{c c_{2}}{(\log R e)^{2}} \alpha\left(\beta T_{(1)}+T_{(2)}\right)
$$

Combining the surface-stress distributions (62) and (65), we see that the total surface-stress distribution for the inner Stokes velocity field at $O\left((1 / \log R e)^{2}\right)$ is

$$
f_{1}(R e) T^{1}=\frac{c}{(\log R e)^{2}}(\gamma-\log 4-c)\left(T_{(1)}+\alpha T_{(2)}\right)+\frac{c c_{2}}{(\log R e)^{2}} \alpha\left(\beta T_{(1)}+T_{(2)}\right)
$$

The complete inner (Stokes') solution through terms of $O(1 / \log R e)$ is thus

$$
\begin{aligned}
u & =f_{0}(R e) u^{0}+f_{1}(R e) u^{1}+\ldots \\
& =f_{0}(R e) u^{0}\left\{1-\frac{1}{\log R e}(\gamma-\log 4-c)\right\}+f_{0}(R e) u_{T}^{0} \frac{c_{2} \alpha}{\log R e}+\ldots,
\end{aligned}
$$

where

$$
u_{T}^{0} \equiv 4 \pi \int_{\Gamma}\left[\left(\log \frac{1}{r}\right) I+\frac{(x-\eta)(x-\eta)}{r^{2}}\right]\left[\beta\left(\tilde{T}_{2 n}\right)_{(1)}+\left(\tilde{T}_{2 n}\right)_{(2)}\right] \mathrm{d} \Gamma
$$

The hydrodynamic force on the cylinder (which includes both the drag and the lift) can be shown readily from the Stokes expansion (68) to be

$$
\begin{aligned}
F & =-f_{0}(R e) \int T^{0} \cdot n \mathrm{~d} \Gamma-f_{1}(R e) \int T^{1} \cdot n \mathrm{~d} \Gamma \\
& =\frac{4 \pi}{-\log R e} e_{1}+\frac{4 \pi}{(\log R e)^{2}}(\gamma-\log 4-c) e_{1}-\frac{4 \pi c \alpha}{(\log R e)^{2}} e_{2}+O\left(\frac{1}{(\log R e)^{3}}\right) .
\end{aligned}
$$


It can be noted that the contribution to the drag force of $O(1 / \log R e)$ is independent of the cylinder geometry, whereas the drag contribution at $O\left((1 / \log R e)^{2}\right)$ contains $c$, which depends on the cylinder geometry. The lift force, which is $O\left((1 / \log R e)^{2}\right)$, includes the two parameters $c$ and $\alpha$, and is also dependent upon the cylinder geometry.

\section{Inertial effects}

Proudman \& Pearson (1957) showed that the first $n$ gauge functions for uniform flow past a circular cylinder are given by

$$
\begin{aligned}
& f_{n}(R e)=\frac{1}{(\log R e)^{n+1}}, \\
& f_{n}(R e)=\frac{1}{(\log R e)^{n}},
\end{aligned}
$$

and it is evident that the same basic result will be true for a cylinder of any arbitrary cross-section. Since the Stokes' solution has a logarithmic singularity at infinity, the inner solution must always be one order higher in $(1 / \log R e)$ in order to match the outer solution. The expansion terms at $O\left((1 / \log R e)^{2}\right)$ that were derived in the preceding section do not include the inertial effects of $O(R e)$ which are asymptotically smaller than terms of $O\left(1 /(\log R e)^{n}\right)$ for any $n$, as $R e \rightarrow 0$. However, we can evaluate the leading-order inertial effects by assuming that the form of the expansion, for small $R e$, is

$$
\begin{aligned}
& (u, p)=\sum_{n=0}^{\infty} \frac{a_{n}}{(\log R e)^{n+1}}(u, p)^{0, n}+\sum_{n=0}^{\infty} \frac{b_{n} R e}{(\log R e)^{n}}(u, p)^{1, n}+\ldots, \\
& (\hat{u}, \hat{p})=\left(e_{1}, 0\right)+\sum_{n=1}^{\infty} \frac{\hat{a}_{n}}{(\log R e)^{n}}(\hat{u}, \hat{p})^{0, n}+\sum_{n=1}^{\infty} \frac{\hat{b}_{n} R e}{(\log R e)^{n}}(\hat{u}, \hat{p})^{1, n}+\ldots
\end{aligned}
$$

The analysis of the preceding section has considered only the first two terms in the first sum in (72) and the first term in the first sum in (73).

When (72) and (73) are substituted into (2) and (9), respectively, we can obtain the following equations for the leading terms.

For the inner expansion,

$$
\begin{aligned}
& 0=-\nabla p^{0, n}+\nabla^{2} u^{0, n}, \text { for all } n, \\
& 0=-\nabla p^{1, n}+\nabla^{2} u^{1, n}, \quad \text { for } n=0 \text { and } 1, \\
& 0=-\nabla p^{1,2}+\nabla^{2} u^{1,2}-\frac{a_{0}^{2}}{b_{2}} u^{0,0} \cdot \nabla u^{0,0} .
\end{aligned}
$$

For the outer expansion, on the other hand,

$$
\begin{aligned}
& 0=-\hat{\nabla} \hat{p}^{0,1}+\hat{\nabla}^{2} \hat{a}^{0,1}-\frac{\partial \hat{a}^{0,1}}{\partial \hat{x}_{1}}, \\
& 0=-\hat{\nabla} \hat{p}^{0,2}+\hat{\nabla}^{2} \hat{a}^{0,2}-\frac{\partial \hat{a}^{0,2}}{\partial \hat{x}_{1}}-\frac{\hat{a}_{1}^{2}}{\hat{a}_{2}} \hat{\boldsymbol{u}}^{0,1} \cdot \boldsymbol{\nabla} \hat{u}^{0,1}, \\
& \mathbf{0}=-\boldsymbol{\nabla} \hat{p}^{1,0}+\hat{\nabla}^{2} \hat{\boldsymbol{u}}^{1,0}-\frac{\partial \hat{\boldsymbol{u}}^{1,0}}{\partial \hat{x}_{1}}, \\
& 0=-\hat{\nabla} \hat{p}^{1,1}+\hat{\nabla}^{2} \boldsymbol{u}^{1,1}-\frac{\partial \boldsymbol{a}^{1,1}}{\partial \hat{x}_{1}}-\frac{\hat{a}_{1} \hat{b}_{0}}{\hat{b}_{1}}\left(\hat{\boldsymbol{u}}^{0,1} \cdot \hat{\nabla} \hat{u}^{1,0}+\hat{a}^{1,0} \cdot \boldsymbol{\nabla} \hat{u}^{0,1}\right) .
\end{aligned}
$$


Thus, in order to calculate the first term in the $O\left(R e /(\log R e)^{n}\right)$ sum in (72), we need know only the first term in the $O\left((1 / \log R e)^{n+1}\right)$ sum. Similarly, to calculate the first two terms in the $O\left(R e /(\log R e)^{n}\right)$ sum of $(73)$, we need to know only $\hat{\boldsymbol{u}}^{\mathbf{0}, 1}$ from the $O\left((1 / \log R e)^{n}\right)$ sum.

It is apparent that the governing equations are either homogeneous Stokes' or Oseen equations, or inhomogeneous versions of these equations. The framework to solve these equations has already been developed in the preceding sections. A solution for the homogeneous equations is either the general Stokes' solution (21) or the general Oseen's solution (37). A particular solution in either case can always be expressed in terms of the fundamental solutions (18-19) and (28-29), respectively. For (76), the appropriate form is

$$
u_{p}^{1,2}=\frac{a_{0}^{2}}{b_{2}} \int_{\Omega}\left[u^{0,0}(\eta) \cdot \nabla u^{0,0}(\eta)\right] \cdot\left[\left(\log \frac{1}{r}\right) I+\frac{(x-\eta)(x-\eta)}{r^{2}}\right] \mathrm{d} \eta,
$$

while for (78) the particular solution takes the form

$$
\hat{u}_{p}^{1,2}=\frac{\hat{a}_{1}^{2}}{\hat{a}_{2}} \int_{\Omega}\left[\hat{u}^{0,1}(\hat{\eta}) \cdot \nabla \hat{u}^{0,1}(\hat{\eta})\right] \cdot \hat{u}^{*}(\hat{x}-\hat{\eta}) \mathrm{d} \hat{\boldsymbol{\eta}},
$$

where $\Omega$ is the flow domain. Note that $\hat{\boldsymbol{u}}^{*}$ is a second-order tensor as given in (32). Applying the same matching procedure as the previous section, we can calculate $b_{2}, u^{1,2}$ which represents the first direct inertial effects in the inner region.

\section{Numerical procedure and results}

In the preceding sections, we have derived the fundamental solutions for twodimensional flow satisfying, respectively, Stokes and Oseen equations, and shown how these may be incorporated into the method of matched asymptotic expansions to provide the basis for numerical, boundary-integral determinations of the effects of weak inertia for uniform two-dimensional flow past arbitrary cylindrical bodies. For the most general case, the leading-order Stokes approximation to the velocity fields, $f_{0}(R e) \boldsymbol{u}^{\mathbf{0}}$, which matches the uniform-flow approximation to the outer solution, is obtained by setting $f_{0}(R e)=1 / \log R e$, solving the integral (44) for the particular cylinder geometry to obtain $T_{(j)}$ for $j=1$ and 2 , and then using $(53 a)$ and $(53 b)$ to obtain $\alpha$ and $c$. The velocity $u^{0}$ is then given in terms of $\tilde{u}_{(\mathrm{j})}$ by (50). The second term, $f_{1}(R e) \boldsymbol{u}^{1}=(1 / \log R e)^{2} u^{1}$, in the inner region can then be evaluated by simply using the same fundamental stress tensors, $T_{(j)}$ for $j=1,2$, to calculate $\beta$ from (65) and $c_{2}$ from (64), With the velocity field given by (68) and the corresponding contributions to the force on the cylinder by (69). If the velocity in the outer region is desired, it can be evaluated directly from (11), (12), (13), (37), (55), (57) and (58).

The equation (44) for $T_{(j)}$ is solved numerically in the present study using a collocation method which transforms the integral equation into a linear system of algebraic equations. The boundary is divided into small elements so that the components of $T_{(f)}$ can be approximated by their value at the centre of each element. This approximation allows us to evaluate the integrals in an element by a simple numerical integration. When $\boldsymbol{x}=\boldsymbol{\eta}$, the integrals obviously become singular. However, integration over a small neighbourhood of $\boldsymbol{x}$ can be achieved analytically by linear expansion of the integrands in (44). When the boundary is divided into $N$ segments, the size of the linear matrix becomes $2 N \times 2 N$ owing to the fact that there are two unknown variables $\tilde{T}_{n x}$ and $\tilde{T}_{n y}$. The linear matrix can be solved straightforwardly by a Gaussian elimination method. 
It should be noted that the boundary integral method reduces the dimensionality of problems by one. Hence, the two-dimensional problems considered in this paper require only the calculation of singularity distribution along line boundaries. This significantly reduces the amounts of computation, compared with a general twodimensional finite-element or finite-difference method.

The total number and size of the boundary elements can be determined from the numerical accuracy required and the variation of the singularity strength along the boundaries. The numerical method is very straightforward and robust in the sense that even a small number of segments still provides a very reasonable answer and the numerical accuracy improves monotonically with the total number of segments.

In the remainder of this section, we demonstrate the utility of the method by applying it to obtain solutions for streaming flow past an elliptic cylinder, a rectangular cylinder, and two parallel circular cylinders which may be either equal in diameter or of two different diameters.

All of these problems, with the exception of two unequal cylinders, were considered by Yano \& Kieda (1980) whose work bears some superficial resemblance to the present study. Yano \& Kieda solved approximately for uniform flows past cylindrical bodies by using fundamental solutions of the Oseen equation. Oseenlet singularities were distributed inside the cylinder(s) and their strength and location were determined by a least-squares method to satisfy approximately the no-slip boundary condition on the cylinder boundary. Of course, the present study is based upon a full matched asymptotic formulation. If we were interested in solving the Oseen equation by itself, however, the boundary-integral technique described in $\$ 3$ could be applied more accurately, without the unnecessary approximations that were introduced by Yano \& Kieda (1980).

\subsection{The elliptic cylinder}

The simplest form of an elliptic cylinder is a circular cylinder. The problem of streaming flow past a circular cylinder at low Reynolds number has been analysed by many prior investigators (cf. Kaplun 1957; Proudman \& Pearson 1957; Van Dyke 1975). We begin here by briefly reconsidering the circular-cylinder problem in the present context. A major simplification is that the coefficient $\alpha$ can be anticipated a priori to have a value of zero, i.e.

$$
\alpha=0 \text {, }
$$

since there is no lift force on a circular cylinder in creeping flow. The fundamental stress distribution on the cylinder can be calculated easily from (44) by using a collocation method. In the numerical calculation, 5-digit accuracy was obtained from 20 uniformly-sized segments along the circular boundary. The numerical result is

$$
\begin{aligned}
& \left(\tilde{T}_{n x}\right)_{(1)}=4.0000 \\
& \left(\tilde{T}_{n y}\right)_{(1)}=0.0000 .
\end{aligned}
$$

From $(53 b), c$ can then be readily evaluated:

$$
c=0.5000,
$$

and the drag force from (69) becomes

$$
F_{x}=-\frac{4.0000 \pi}{\log R e}\left[1+\frac{1}{\log R e}\left(\frac{1}{2}-\gamma+\log 4\right)+\ldots\right] .
$$




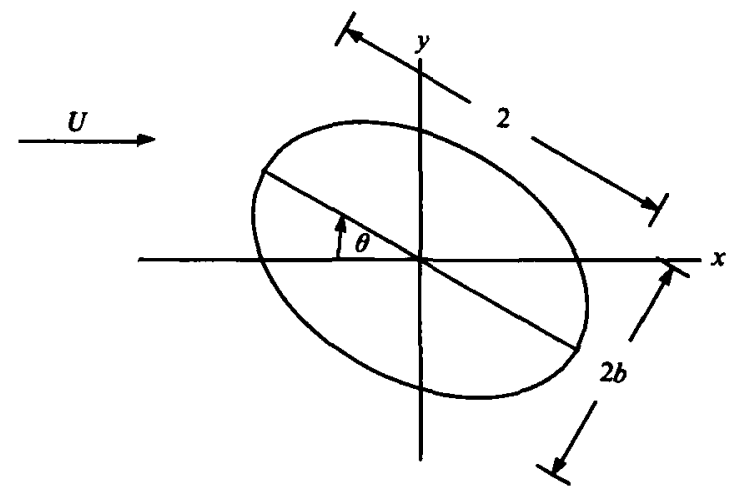

FrgurR 1. An inclined elliptic cylinder.

Not surprisingly, this expression for the drag force is identical with the results of Proudman \& Pearson (1957).

Let us now turn to the case when the cylinder has an elliptic cross-section, and is inclined at an arbitrary angle relative to the streaming velocity field upstream. $A$ schematic diagram is given in figure 1. The lengths of the two principal semi-axes are respectively 1 and $b$, (in effect, we have non-dimensionalized using the length of the major semi-axes), and the angle of inclination is denoted by $\theta$. The inclined cylinder will experience a lift force as well as a drag force except when $\theta=0$ and $\frac{1}{2} \pi$. This elliptic-cylinder problem was, in fact, previously solved analytically by Shintani, Umemura \& Takano (1983) using the method of matched asymptotic expansions and an elliptical cylindrical coordinate system. Comparison of our results with those of Shintani et al. (1983) thus provides a further basis for assessing the numerical accuracy and fundamental correctness of our present technique.

Our prescription for solution, for any given $\theta$, is to first solve (44) to obtain $T_{(1)}$ and $T_{(2)}$, and then use these stress distributions to obtain the constants $c$ and $\alpha$ from $(53 a)$ and $(53 b)$, and the second-order constants $\beta$ and $c_{2}$ from (64) and (65). When the geometry is changed only by changing the angle of inclination, $\theta$, relative to the uniform flow, without altering the cross-sectional geometry of the cylinder, however, one need only determine the surface-stress distribution for motion of the cylinder in any two orthogonal directions to determine the solution for motion with any arbitrary angle of inclination. In the present case of an elliptic cylinder, it is convenient to solve for motions parallel to the two principal axes of the ellipse. These two problems correspond to motion in the $x$-and $y$-directions of an elliptic cylinder whose major principal axis lies in the $x$-coordinate direction (i.e. $\theta=0$ ). With these two problems solved, we can evaluate the drag forces, $G_{1}$ and $G_{2}$, corresponding to creeping motion along the $x$-coordinate and $y$-coordinate, respectively:

$$
G_{1} \equiv \int_{\Gamma} e_{1} \cdot\left[T_{(1)}(\theta=0) \cdot n\right] \mathrm{d} \Gamma
$$

and

$$
G_{2} \equiv \int_{\Gamma} e_{2} \cdot\left[T_{(2)}(\theta=0) \cdot n\right] \mathrm{d} \Gamma
$$

Now, the force on an elliptic cylinder with arbitrary angle of inclination, $\theta$, can be expressed in terms of $G_{1}$ and $G_{2}$. In particular, if we denote the force in the $j$-direction 


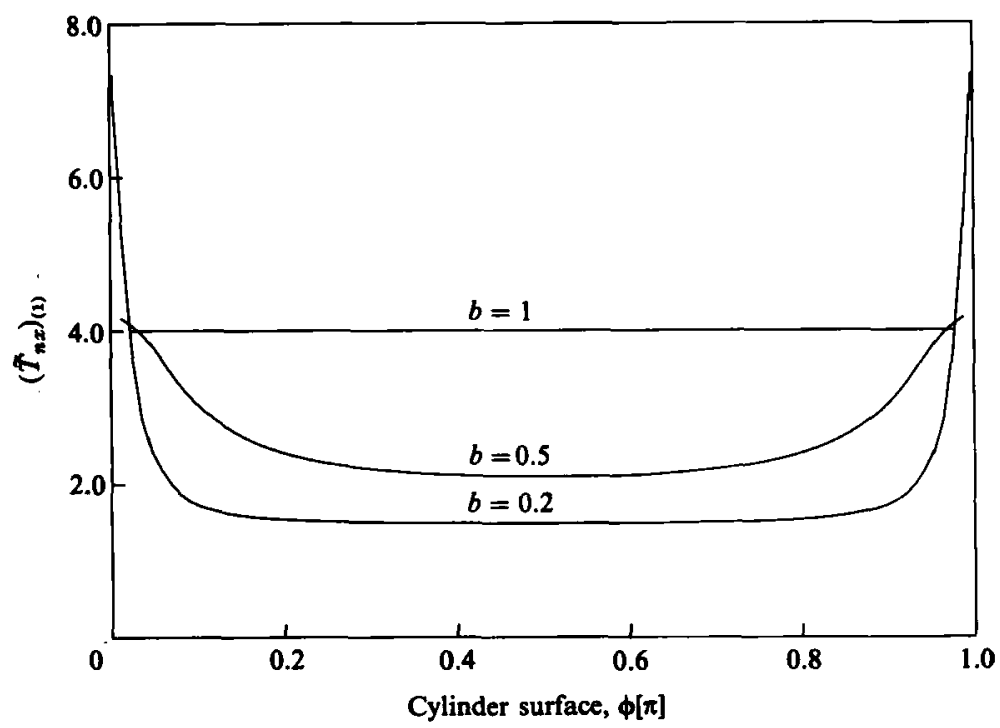

FIGURE 2. $\left(\tilde{T}_{n x}\right)_{(1)}$ on the elliptic cylinder with $\theta=0$.

due to creeping motion in the $i$-direction as $H_{i j}$, it follows from the linearity of the problem and simple geometric considerations that

$$
\begin{aligned}
& H_{x x}=G_{1} \cos ^{2} \theta+G_{2} \sin ^{2} \theta, \\
& H_{y y}=G_{1} \sin ^{2} \theta+G_{2} \cos ^{2} \theta, \\
& H_{x y}=\frac{1}{2}\left(G_{2}-G_{1}\right) \sin 2 \theta, \\
& H_{y x}=\frac{1}{2}\left(G_{2}-G_{1}\right) \sin 2 \theta .
\end{aligned}
$$

The fundamental stress tensor, $T_{(j)}(\theta)$, corresponding to translation in the $j$-direction $\left(j=1\right.$ or 2 ) with an arbitrary, fixed inclination angle $\theta$, is related to the $H_{i j}$ according to

$$
H_{i j}=\int_{\Gamma} e_{i} \cdot\left(\boldsymbol{T}_{j}(\theta) \cdot n\right) \mathrm{d} \Gamma .
$$

It follows from (53a) that $\alpha$ is given by

$$
\alpha=-\frac{H_{x y}}{H_{y y}},
$$

while the coefficient $c$ can be shown, from $(53 b)$, to be

$$
c=\frac{4 \pi}{H_{x x}+\alpha H_{y x}}=\frac{4 \pi H_{y y}}{H_{x x} H_{y y}-H_{x y} H_{y x}} .
$$

Then the drag force and lift force in (69) becomes

$$
\begin{aligned}
& F_{x}=-\frac{4 \pi}{\log R e}+\frac{4 \pi}{(\log R e)^{2}}\left(\gamma-\log 4-\frac{4 \pi H_{y y}}{H_{x x} H_{y y}-H_{x y} H_{y x}}\right)+O\left(\frac{1}{(\log R e)^{3}}\right), \\
& F_{y}=-\frac{(4 \pi)^{2}}{(\log R e)^{2}} \frac{H_{x y}}{H_{x x} H_{y y}-H_{x y} H_{y x}}+O\left(\frac{1}{(\log R e)^{3}}\right) .
\end{aligned}
$$




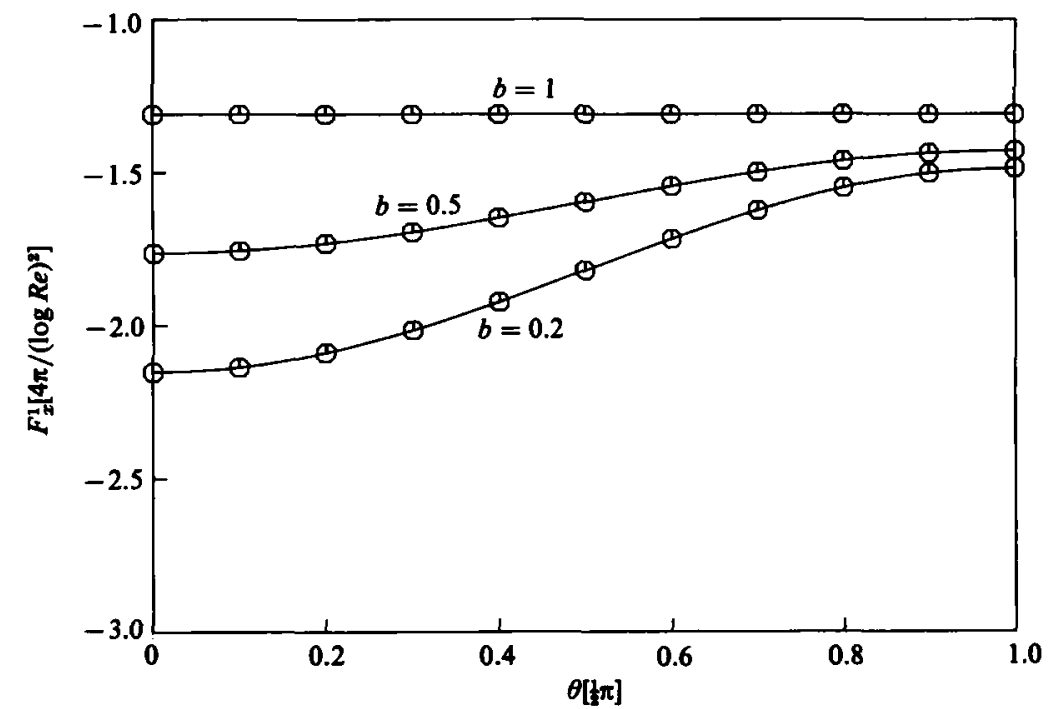

Figure 3. $F_{x}^{1}$ on the elliptic cylinder; $\bigcirc$, from Shintani et al. (1983); - calculated.

To proceed further, it is necessary to solve the integral (44) for the surface-stress distributions, $T_{(1)}(\theta=0)$ and $T_{(2)}(\theta=0)$ for motion in the $x$ - and $y$-directions, respectively. Once $T_{(j)}(\theta=0)$ is known for $j=1$ and 2 , the values of $G_{1}$ and $G_{2}$ can be determined, whence $H_{i j}$ and the contributions to the force at $O\left((1 / \log R e)^{2}\right)$ for an arbitrary angle of inclination.

We apply a collocation method to numerically evaluate $\boldsymbol{T}_{(j)}$ on the elliptic cylinder. In figure 2, the distribution of $\left(T_{n x}\right)_{(1)}$ is plotted for the cases of $b=1,0.5$ and 0.2 . As the elliptic cylinder gets thinner (i.e. $b$ becomes smaller), the stress increases rapidly around the edges. In these cases we employed smaller segments around the edges to ensure accuracy in the numerical results.

The contributions to the force on the cylinder at $O\left((1 / \log R e)^{2}\right)$, i.e. $F^{1}$, have been calculated for $b=0.2,0.5$ and 1 , using (92) and (93). The results for the drag $F_{x}^{1}$ and the lift $F_{y}^{1}$ are plotted in figures 3 and 4 , respectively, as a function of the angle of inclination $\theta$. The numerically calculated values are in excellent quantitative agreement with the analytical results of Shintani et al. (1983) which are also plotted in figures 3 and 4 . For $b \leqslant 1, F_{x}^{1}$ decreases as the aspect ratio $b$ becomes smaller and increases as the angle of inclination $\theta$ increases. As depicted in figure 4, the cylinder experiences an increased lift force $F_{y}^{1}$ as the cylinder gets thinner. The lift reaches a maximum value at $\theta=\frac{1}{4} \pi$.

The problem of flow past an elliptic cylinder can, of course, be solved by analytical methods, as we have seen already, using elliptical cylindrical coordinates (Shintani et al. 1983). The present boundary-integral approach can be applied equally well, however, to problems involving more complicated geometry. For example, in the next section we consider the flow past a rectangular cylinder which is not accessible to an analytic approach with any reasonable level of effort.

\subsection{A rectangular cylinder}

A schematic diagram of the inclined rectangular cylinder is shown in figure 5 . The problem of uniform flow past a rectangular cylinder can be analysed by a very similar scheme to that used in the previous case of an elliptic cylinder. Obviously the zero-order 


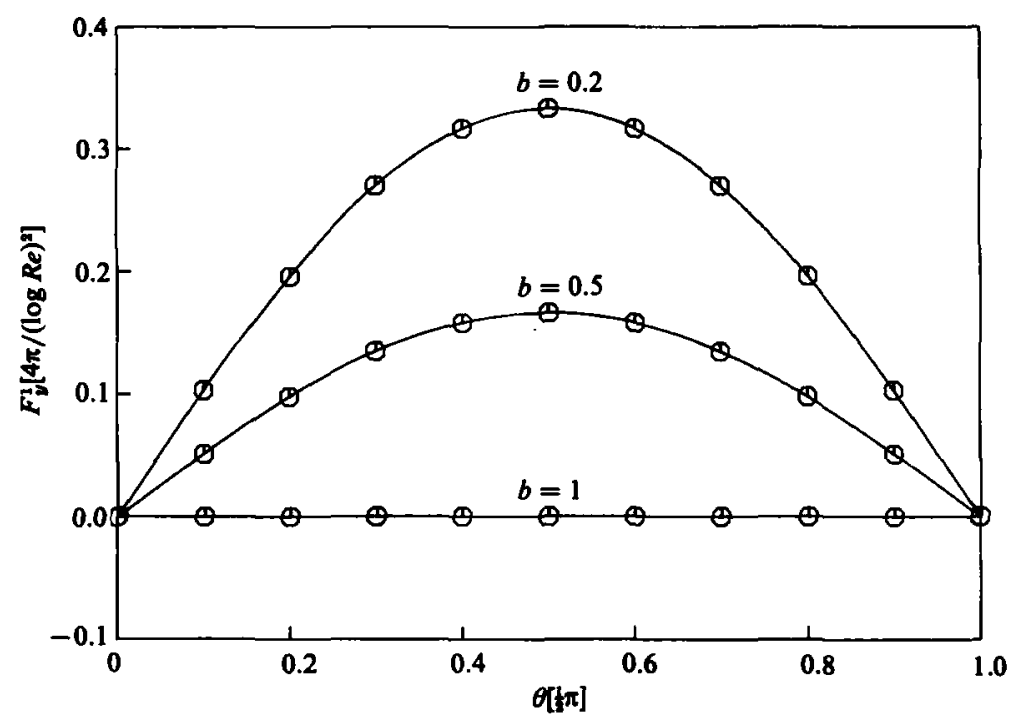

Fiqure 4. $F_{y}^{1}$ on the elliptic cylinder; $O$, from Shintani et al. $(1983) ;-$, calculated.

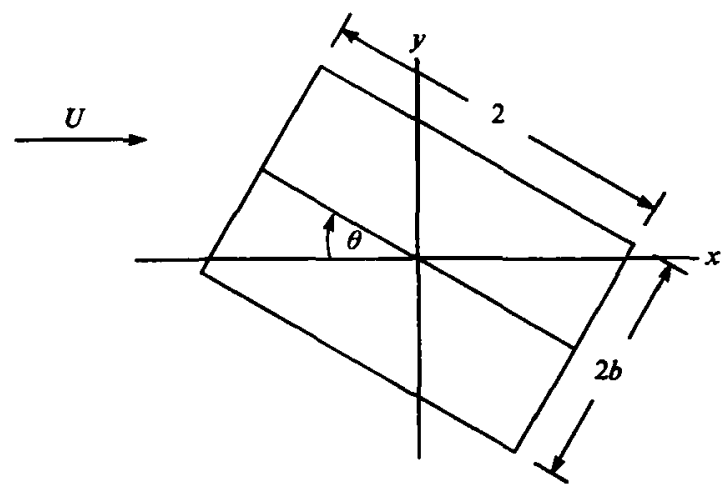

Fiaure 5. An inclined rectangular cylinder.

hydrodynamic drag and lift forces, $F^{0}$, are independent of cylinder geometry. The first-order solution at $O\left((1 / \log R e)^{2}\right)$ contains $H_{i j}$ and thus depends on cylinder geometry. To evaluate $G_{1}$ and $G_{2},(44)$ should be solved by a collocation method. Since the rectangular cylinder contains singular points at the four corners, the stress distribution around the corners was examined carefully by imposing more grid points. As a grid centre is closer to a corner, the calculated stress increases accordingly. However, the overall stress contribution to the drag or lift force converges asymptotically to a finite value as the grid sizes become smaller. For this problem, we used 80 non-uniformly sized segments along the rectangle.

The numerically calculated values of $F_{x}^{1}$ and $F_{y}^{1}$ for $b=0.2,0.5$ and 1 are plotted, respectively, in figures 6 and 7 as a function of the angle of inclination. Clearly, there is a very considerable similarity between the present results and those given earlier for a cylinder of elliptic cross-section. 


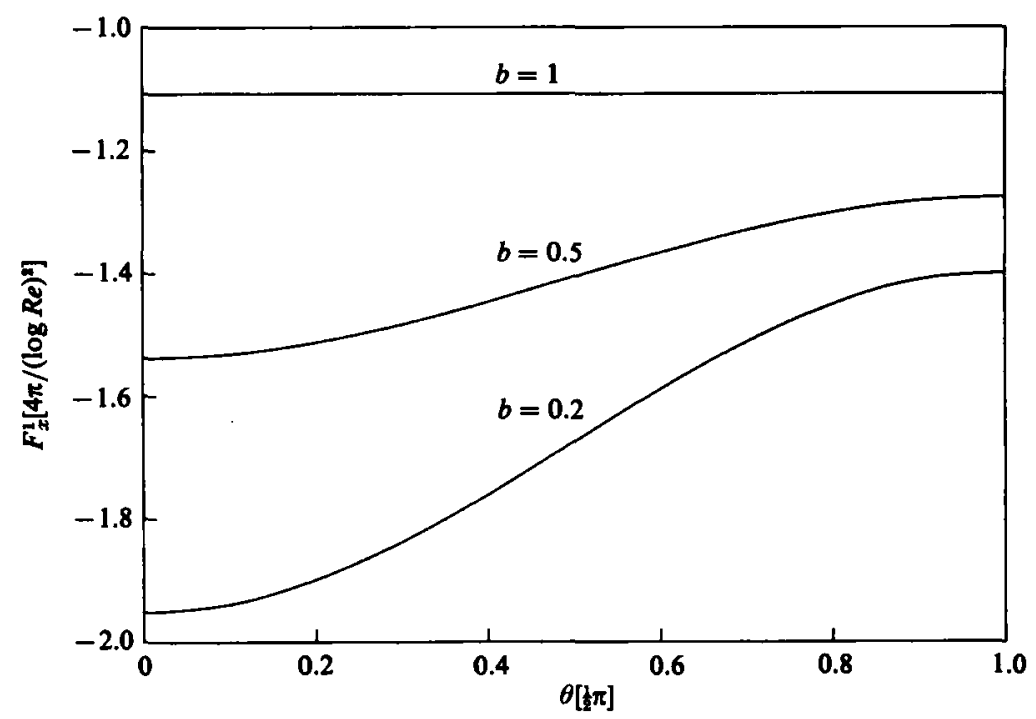

Figure 6. $F_{x}^{1}$ on the rectangular cylinder.

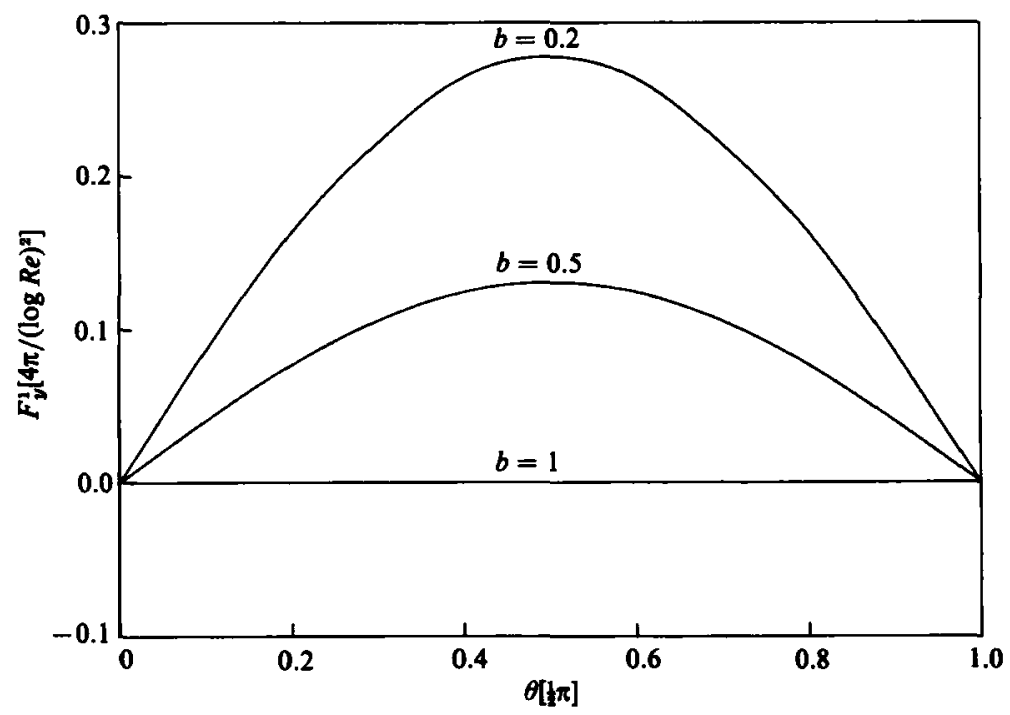

FIQURE 7. $F_{y}^{1}$ on the rectangular cylinder.

\subsection{Two circular cylinders}

The results and derivations in the previous sections were based on the problem of a uniform flow past a single cylindrical body. However, the method can also be extended easily to consider uniform two-dimensional flow past multiple cylindrical bodies. Here, we consider the problem of uniform flow past a pair of circular cylinders as an illustration of the application to multiple cylindrical bodies.

Umemura (1982) investigated uniform flow past two equal circular cylinders by use of bipolar coordinates. He recognized that the general solution of Stokes' equation in bipolar coordinates by Jeffery (1922) yields zero drag force for a cylindrical body in a uniform flow. Hence, in order to correct this non-physical property of the 


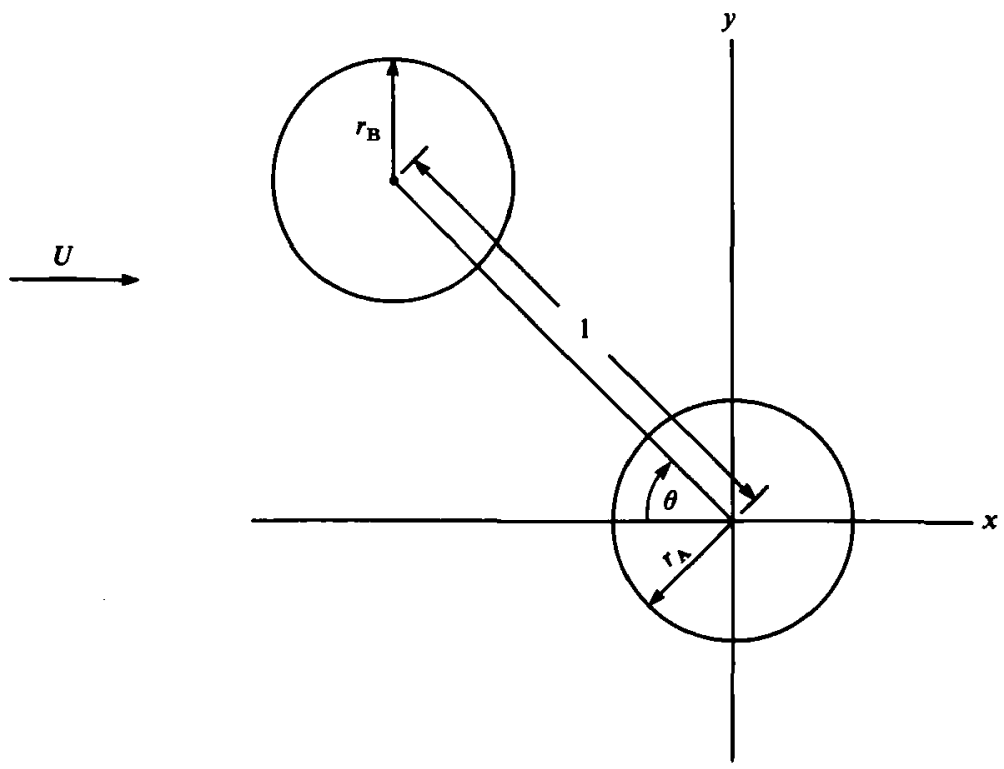

Figure 8. Two circular cylinders.

solution, Umemura added the fundamental solution due to a point force singularity onto Jeffery's solution. Unfortunately, the resulting solution became so complicated that Umemura (1982) arbitrarily abandoned the matching conditions for the particular solution from Oseen's equation. In contrast to this bipolar-coordinate approach, our method can be applied to a multiple-cylinder system just as easily as to a single cylinder.

The method developed in this paper does not have any intrinsic restrictions on the location of separate boundaries. If the distance separating the two cylinders is $>1 / R e$, the general Oseen's solution has to be applied with two cylinder boundaries and then matched with an inner Stokes' solution for each cylinder considered separately. In the present paper, however, we considered, for simplicity, the numerical example in which the two cylinders are always located sufficiently close to each other that the Stokes' flow region encompasses both cylinders.

In figure 8 we show a schematic of the two-cylinder problem. The distance between the cylinder centres is chosen as the characteristic lengthscale. The cylinder located at the origin of the coordinates is denoted as Cylinder $A$ and the other as Cylinder $B$. The dimensionless radii of the two cylinders are $r_{A}$ and $r_{B}$, respectively, and the inclination angle of the line of centres between the cylinders relative to the uniformflow direction is given as $\theta$. Note that the two cylinders touch when $r_{A}+r_{B}=1$.

As discussed previously, the linearity of Stokes' equation allows us to superpose solutions for the two fundamental cases, $\theta=0$ and $\theta=\frac{1}{2} \pi$, to generate the flow field around two cylinders with an arbitrary angle of inclination, $\theta$.

A multiple-cylinder system can be considered as a 'generalized' cylindrical body whose surface is disconnected or discontinuous. Consequently, (69) can still be used to calculate the 'total' hydrodynamic force on the multiple-cylinder system (i.e. the sum of the hydrodynamic forces that act on the individual cylinders). Let us follow the nomenclature originally introduced for the elliptic-cylinder problem. Thus, we denote the total hydrodynamic forces for the fundamental creeping-flow problems (44) with $\theta=0$ and $\theta=\frac{1}{2} \pi$ as $G_{1}$ and $G_{2}$. The total hydrodynamic force in the $j$-direction 


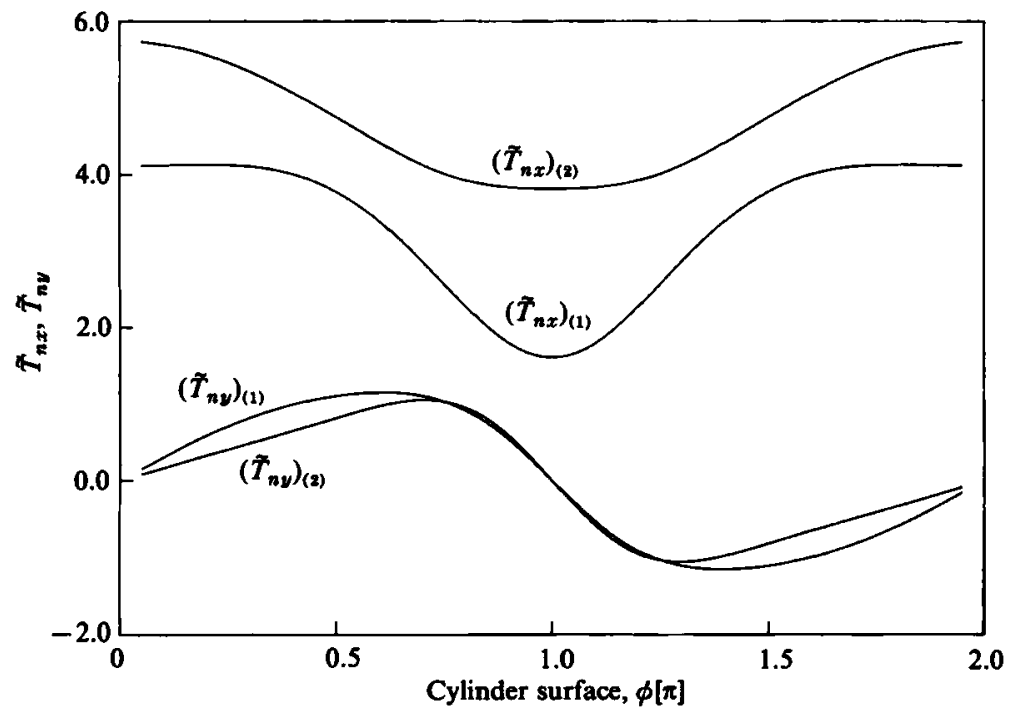

Figure 9. Stress distribution on Cylinder $\mathrm{A} ; r_{\mathrm{A}}=r_{\mathrm{B}}=\mathbf{0 . 2 5}$.

due to motion in the $i$-direction with an arbitrary angle of inclination $\theta$, is then denoted as $H_{i j}$ and the total hydrodynamic force corresponding to (69) is $F_{i}$. The individual force components on either cylinder are indicated by means of the superscripts $A$ and $B$, respectively. Thus,

$$
\begin{aligned}
G_{i} & =G_{i}^{\mathrm{A}}+G_{i}^{\mathrm{B}}, \\
H_{i j} & =H_{i j}^{\mathrm{A}}+H_{i j}^{\mathrm{B}}, \\
F_{i} & =F_{i}^{\mathrm{A}}+F_{i}^{\mathrm{B}} .
\end{aligned}
$$

We may note that the leading-order contribution to the total force component $F_{x}$ is still given by $-4 \pi /(\log R e)$ (recall that the leading-order term is independent of geometry) while the total lift component $F_{y}$ is still zero to leading order. Of course, the individual force components $F_{x}$ and $F_{y}$ on either cylinder A or B can vary depending on the geometrical location and sizes of the cylinders. For the $l$ th cylindrical body, the hydrodynamical force can be expressed as

$$
\begin{array}{r}
F^{l}=\frac{-c}{\log R e} \int\left(T_{1}^{0, l}+\alpha T_{2}^{0, l}\right) \cdot n \mathrm{~d} \Gamma+\frac{c}{(\log R e)^{2}}(\gamma-\log 4-c) \int\left(T_{1}^{0, l}+\alpha T_{2}^{0, l}\right) \cdot n \mathrm{~d} \Gamma \\
-\frac{c c_{2}}{(\log R e)^{2}} \alpha \int\left(\beta T_{1}^{0, l}+T_{2}^{0, l}\right) \cdot n \mathrm{~d} \Gamma+O\left(\frac{1}{(\log R e)^{3}}\right) .
\end{array}
$$

Note that $c, c_{2}, \alpha$ and $\beta$ are still determined by the total drag and lift forces on the two-cylinder system. Although the zero-order lift force for the total 'composite' cylindrical 'body' is identically zero, as we have already noted, an individual cylinder can experience a non-zero lift force.

\subsection{Two equal cylinders}

Let us first consider uniform flow past two equal cylinders. In this case, the two cylinders experience identical drag and lift forces in the low-Reynolds-number limit. In figure 9 we show the stress distributions, $\tilde{T}_{n x}$ and $\tilde{T}_{n y}$, along the cylinder surface 


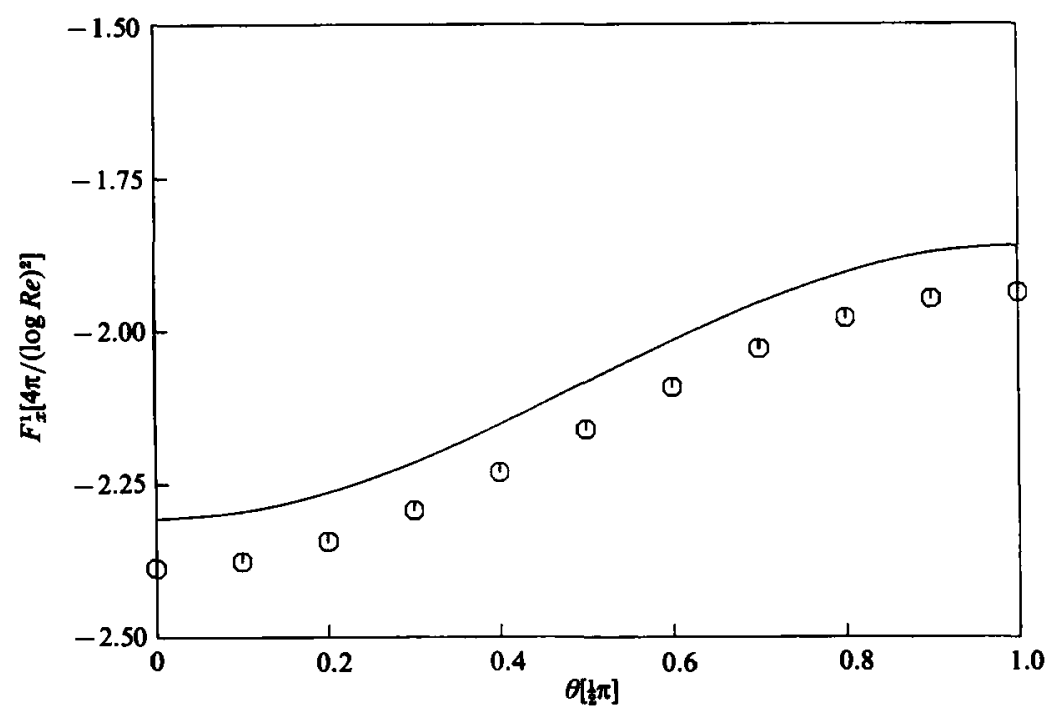

Figure 10. $F_{x}^{1}$ on the two cylinders $\left(F_{x}^{1}=F_{x}^{1 \mathrm{~A}}+F_{x}^{1 \mathrm{~B}}, r_{\mathrm{A}}=r_{\mathrm{B}}=0.25\right)$; $\mathrm{O}$, from Umemura (1980); ——, calculated.

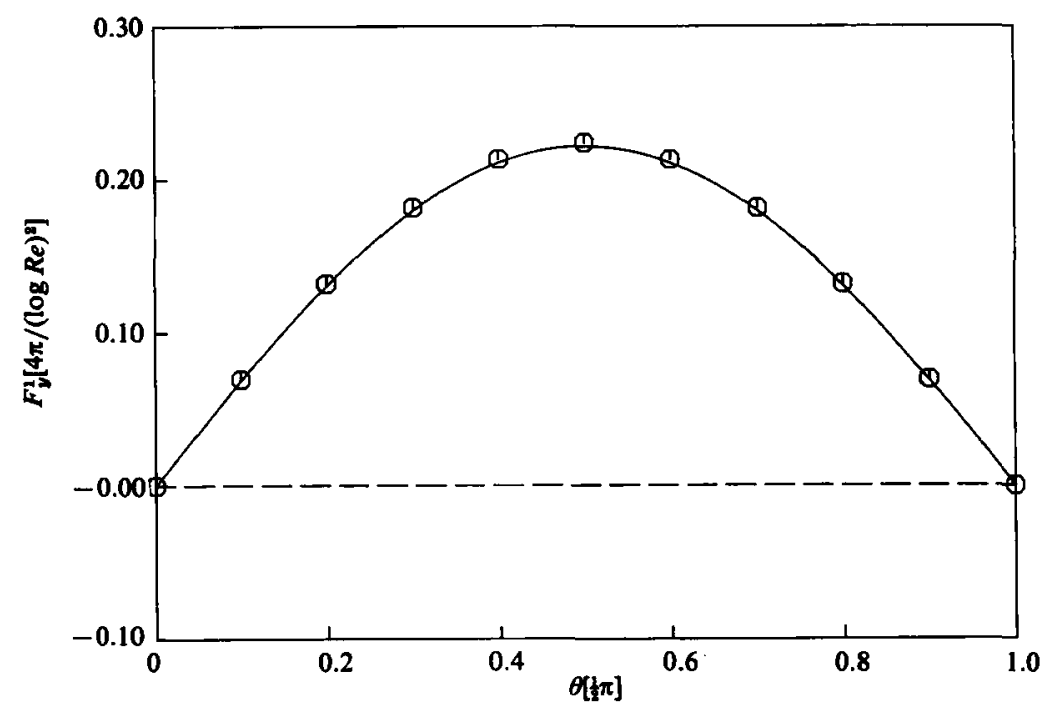

Fiaure 11. $F_{y}^{1}$ on the two cylinders $\left(F_{y}^{1}=F_{y}^{1 \mathrm{~A}}+F_{y}^{\mathrm{BB}}, r_{\mathrm{A}}=r_{\mathrm{B}}=0.25\right)$; $\mathrm{O}$, from Umemura (1980); - , calculated.

for the case of $r_{\mathrm{A}}=r_{\mathrm{B}}=0.25$. The presence of the second cylinder creates strong non-uniformities in $\widetilde{T}_{n x}$ and $\widetilde{T}_{n x}$. Note that, for a single circular cylinder, a uniform flow produces constant values for $\left(\widetilde{T}_{n x}\right)_{(1)}$ and $\left.\left(\tilde{T}_{n y}\right)_{(1)}\left(\left(\tilde{T}_{n x}\right)_{(1)}=4,\left(\tilde{T}_{n y}\right)_{(1)}=0\right)\right)$ along the cylinder surface.

When the line of centres connecting the two cylinders is rotated through an angle $\theta$, the zero-order solution for total drag and lift forces is still the same as for the case of a single cylinder. The first-order contributions, $F_{x}^{1}$ and $F_{y}^{1}$, are compared with Umemura's (1982) results in figures 10 and 11. Umemura used the Oseen's solution for a single cylinder in the outer domain and arbitrarily neglected the inhomogeneous 


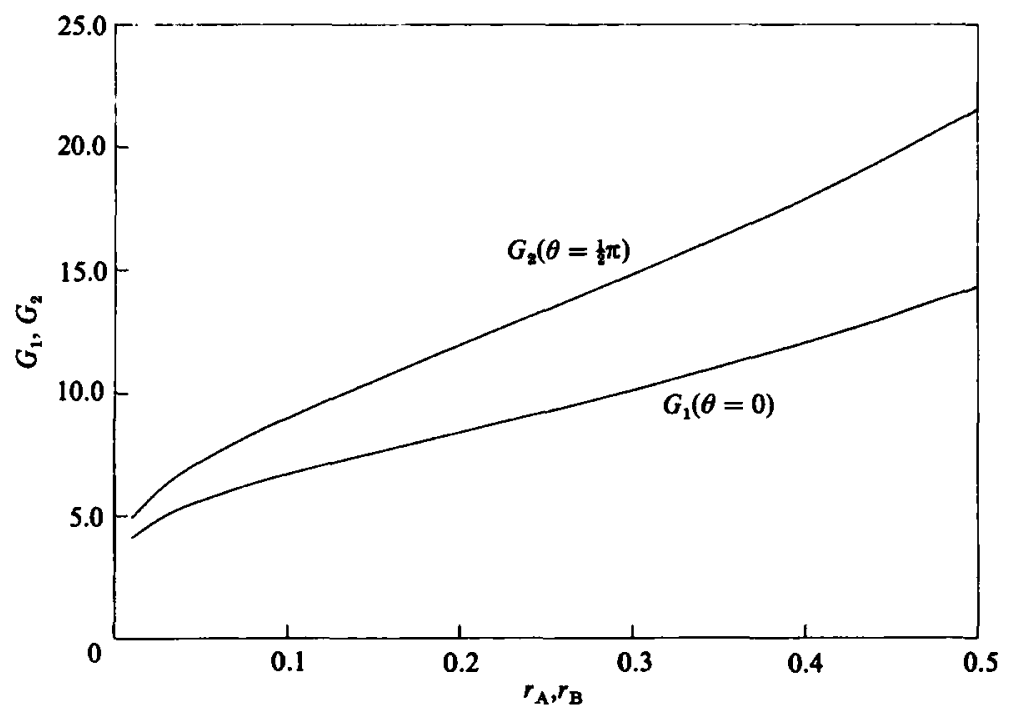

Figure 12. Stokes drag $G_{1}$ and $G_{2} v s$. cylinder size; $r_{\mathrm{A}}=r_{\mathrm{B}}, G_{1}=G_{1}^{\mathrm{A}}+G_{1}^{\mathrm{B}}, G_{2}=G_{2}^{\mathrm{A}}+G_{2}^{\mathrm{B}}$.

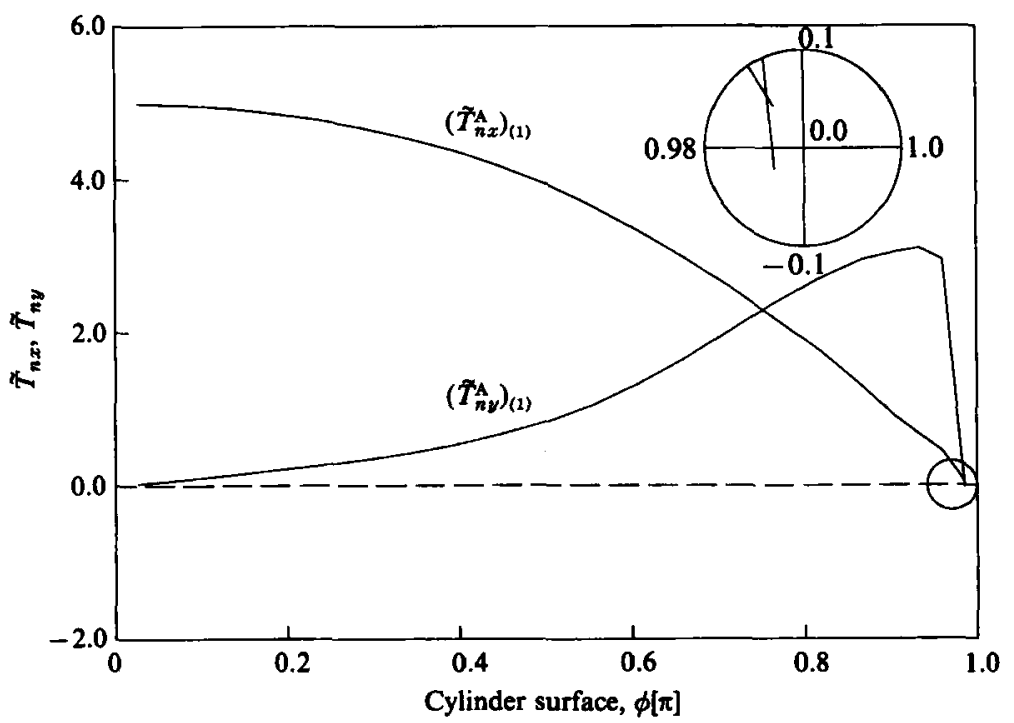

FIGURE 13. Stress distribution on cylinder $A ; r_{A}=r_{B}=0.5, \theta=\frac{1}{2} \pi$.

terms in a higher-order approximation. Nevertheless, his results are still in good agreement with the present calculations. The figures indicate that the lift force $F_{y}^{\prime}$ reaches a maximum when $\theta=\frac{1}{4} \pi$, whereas the drag force $F_{x}\left(\approx F_{x}^{0}\right.$ (constant) $\left.+F_{x}^{\prime}\right)$ monotonically increases with $\theta$.

Since the distance between the two cylinder centres has been chosen as the characteristic lengthscale, the effects of the distance between the two cylinders on the stress can be investigated by changing the radii $r_{\mathrm{A}}$ and $r_{\mathrm{B}}$. In figure 12, the $G_{1}$ and $G_{2}$ are shown for various values of $r_{\mathrm{A}}$ and $r_{\mathrm{B}}$. Evidently, $G_{1}$ and $G_{2}$ become smaller as the radii of cylinders are reduced relative to the distance between centres. 


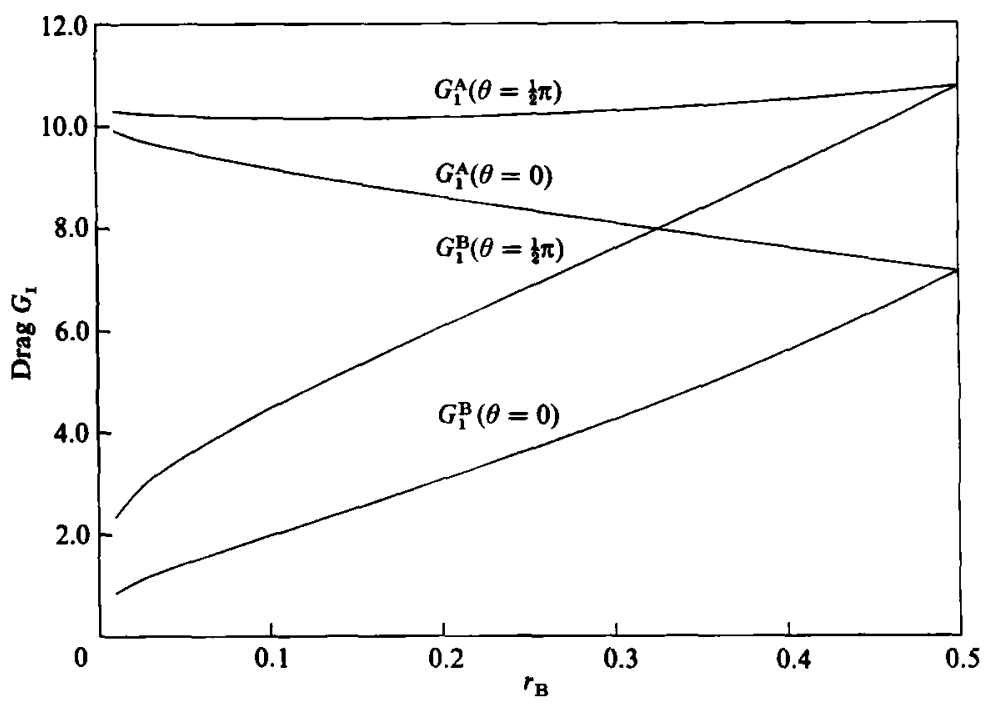

Figure 14. Stokes drag $G_{1} v s$. Cylinder B radius; $r_{\mathrm{A}}=0.5$.

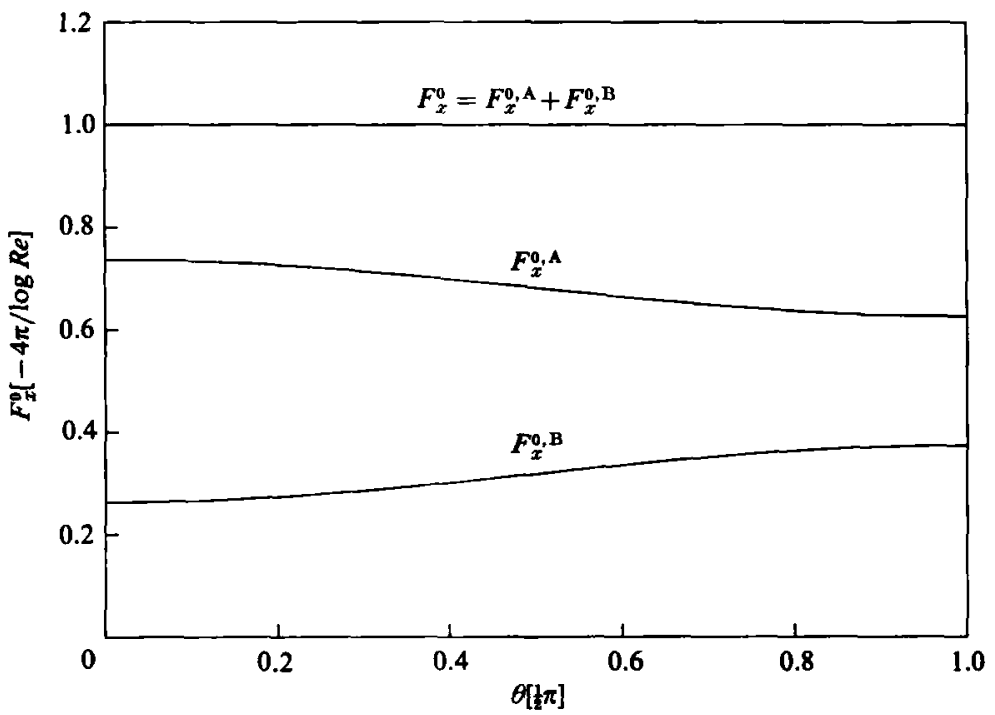

FiguRe 15. $F_{x}^{0}$ on cylinders; $r_{\mathrm{A}}=0.5, r_{\mathrm{B}}=0.2$.

Note, when $r_{A}=r_{B}=0.5$, the cylinders are touching. The drag force for the case of $\theta=\frac{1}{2} \pi$ is always higher than for $\theta=0$ (i.e. $G_{2}>G_{1}$ ).

When the two cylinders are very close to each other or in contact, Moffat's eddies can exist near the corner (Moffat 1964). Figure 13 shows the stress $\left(\tilde{T}_{n x}\right)_{(1)}$ and $\left(\tilde{T}_{n y}\right)_{(1)}$ for $r_{\mathrm{A}}=r_{\mathrm{B}}=0.5$ and $\theta=\frac{1}{2} \pi$. It indicates the presence of flow separation in the corner, corresponding to the existence of weakly negative values for $\left(\tilde{T}_{n x}\right)_{(1)}$ and $\left(\tilde{T}_{n y}\right)_{(1)}$ near the contact points. Dorrepaal \& O'Neil (1979) found several eddies around the contact point. However, since there exists a strong damping effect and the ratio of the intensities of successive eddies is greater than 300 , any experimental observation of a sequence of eddies will be extremely difficult (Moffat 1964).

Since the eddy intensity is very weak, a direct application of a collocation method 


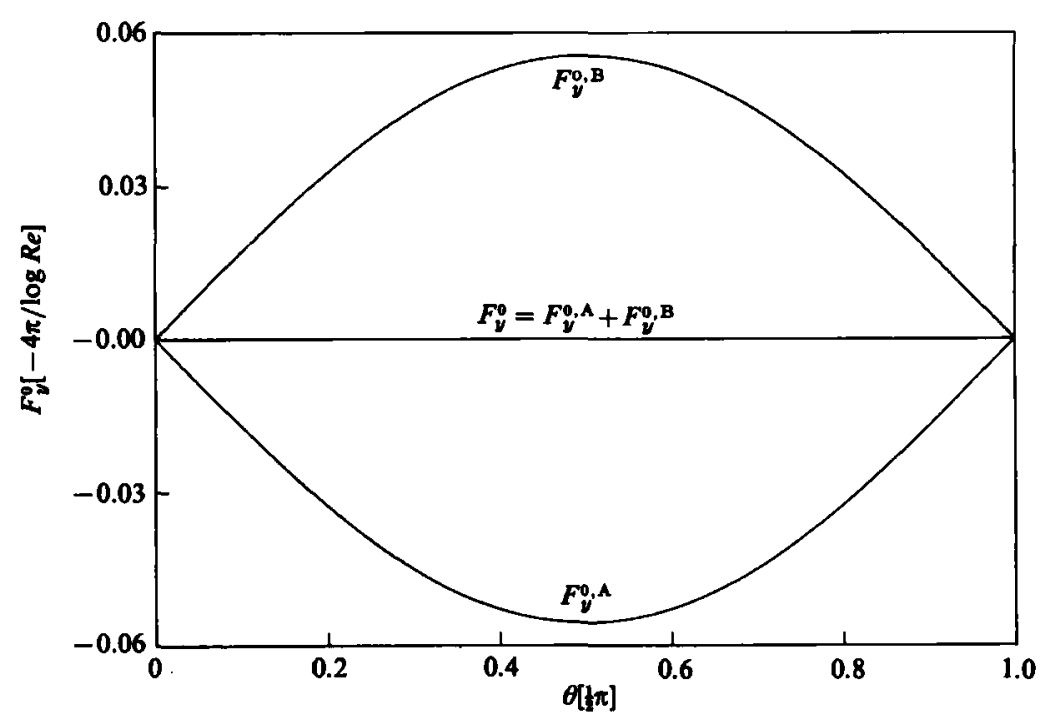

Fiaure 16. $F_{y}^{0}$ on two cylinders; $r_{A}=0.5, r_{B}=0.2$.

is likely to fail to identify the multiple Moffat eddies. Smaller grids around the contact point will make the matrix from a collocation method singular. Nevertheless, since the weak eddies do not affect the overall flow field, it is clear that they could be examined separately with a more elaborate numerical scheme. For present purposes, it is probably superfluous to evaluate the very weak multiple eddies, which will not contribute significantly to the overall flow field or to the drag and lift forces, even though the esoteric multiple eddies are of great mathematical interest.

\subsection{Two unequal cylinders}

Finally, we consider the case of uniform flow past two unequal cylinders. The flow in this case generates non-zero lift forces on the individual cylinders, even in the zero-order solution, and this case deserves to be examined more carefully. In the present numerical example, we fix the radius of the first cylinder at $r_{\mathbf{A}}=0.5$ and vary the radius of the second cylinder between 0 and 0.5 .

The dependence of $G_{1}$ and $G_{2}$ on $r_{\mathrm{B}}$ is plotted in figure 14. We examine the case of $r_{\mathrm{A}}=0.5$ and $r_{\mathrm{B}}=0.2$ more thoroughly to illuminate the problem of non-equal cylinders.

In figures 15 and 16 , we plot $F_{x}^{0}$ and $F_{y}^{0}$ for the individual cylinders vs. the angle of inclination. Although the two cylinders experience unequal drag and lift forces in the zero-order solution, the total drag force always remains constant, equal to $-4 \pi / \log R e$, independent of the body geometry. Similarly, the overall lift force from the zero-order solution is zero, though the unequal-sized cylinders experience a non-zero lift force individually. We also plot $F_{x}^{1}$ and $F_{y}^{1}$ from the first-order solution in figures 17 and 18, which shows the uneven distribution of drag and lift forces between the two cylinders, as a consequence of their differences in size. It is interesting to note that the leading-order contribution to the lift forces $F_{y}^{0, \text { A }}$ and $F_{y}^{0, \text { B }}$ is in the direction to push the cylinders apart. However, the first-order contributions $F_{y}^{1, \mathrm{~A}}$ and $F_{y}^{1, \mathrm{~B}}$ are in the opposite direction, and this reduces the zero-order tendency to push the cylinders apart. 


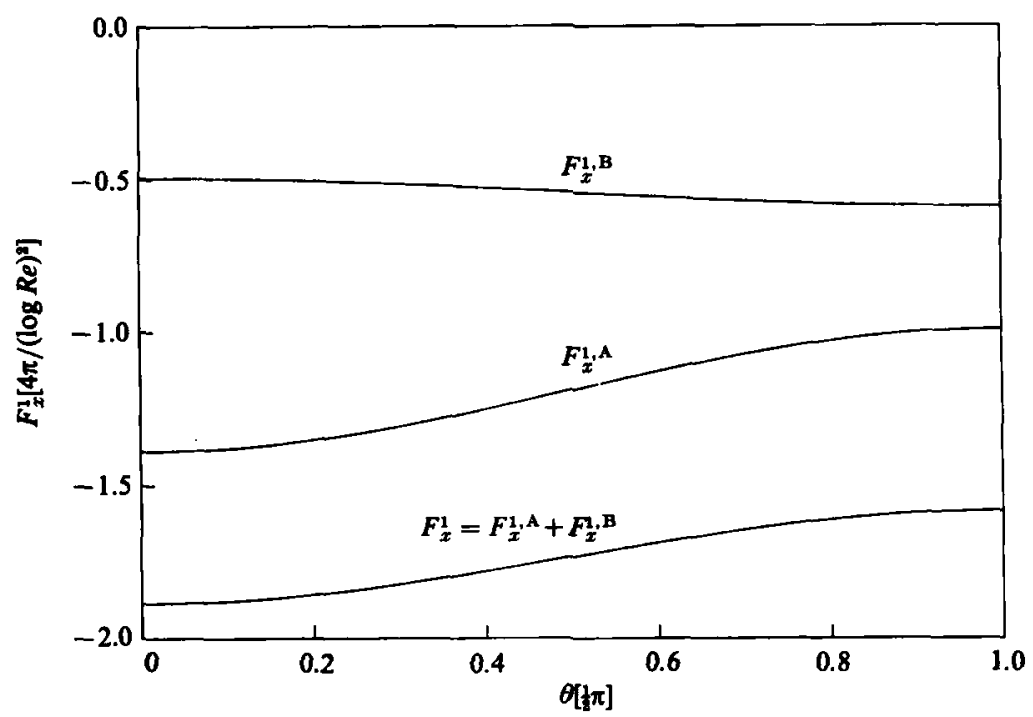

Figure 17. $F_{x}^{\prime \prime}$ on two cylinders; $r_{\mathrm{A}}=0.5, r_{\mathrm{B}}=0.2$.

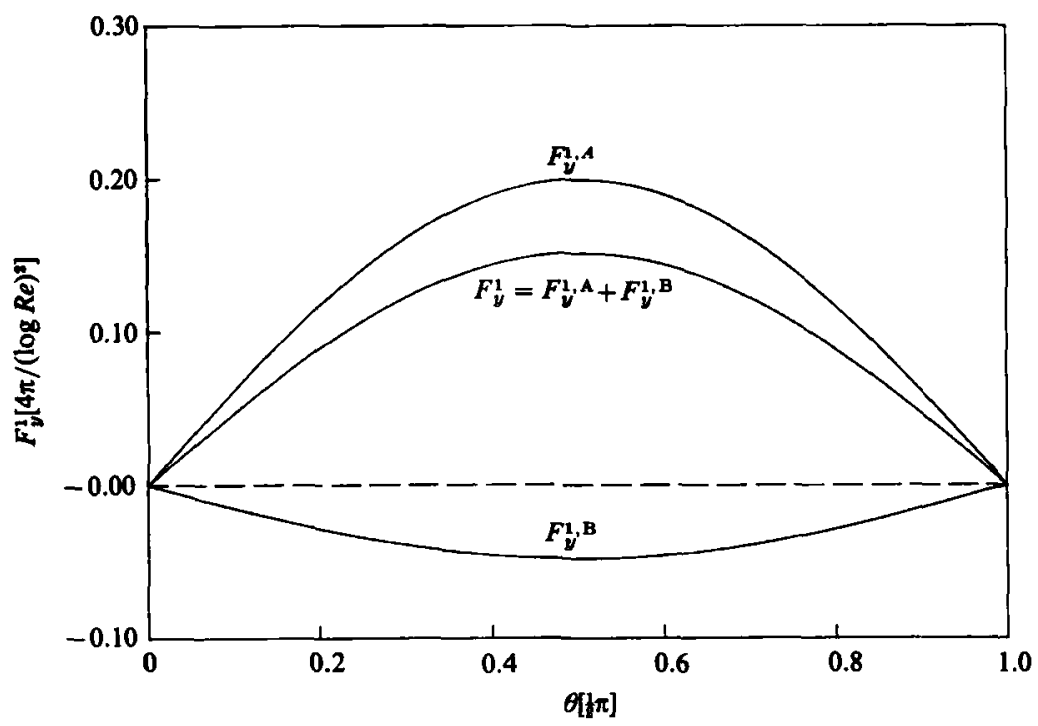

FigURe 18. $F_{y}^{1}$ on two cylinders; $r_{\mathrm{A}}=0.5, r_{\mathrm{B}}=0.2$.

\section{Conclusions}

A numerical method has been developed to calculate low-Reynolds-number flow past an arbitrary cylinder. The solutions for both the inner and outer fields are expressed in terms of integral equations of the second kind. These integral equations are then solved numerically by a collocation method, which takes account of the matching conditions between the outer and inner solutions.

The general solution of Stokes' equation for two-dimensional flow exhibits a logarithmic singularity at infinity, which signals the fact that the Stokes' approximation is not valid except within a distance less than $O(1 / R e)$ from the cylinder. Kaplun (1957) and Proudman \& Pearson (1957) devised a singular-perturbation method which 
asymptotically matches the inner Stokes' solution with a solution of the Oseen equation which is valid in the outer region. In the same spirit, we propose a numerical method of implementing singular-perturbation theory, which can be applied easily for a uniform flow past one (or more) arbitrary cylinders.

For numerical examples, we consider uniform flow past an elliptic cylinder, uniform flow past a rectangular cylinder, and uniform flow past a pair of circular cylinders.

\section{REFERENCES}

Chang, I.-D. \& Fins, R. 1961 On the solutions of a class of equations occurring in continuum mechanics with application to the Stokes paradox. Arch. Rat. Mech. Anal. 7, 388.

DorrepaAt, J. M. \& O'Neir, M. E. 1979 The existence of free eddies in a streaming Stokes flow. Q. J. Mech. Appl. Maths 32, 95.

Fins, R. 1959 On steady-state solutions of the Navier-Stokes partial differential equations. Arch. Rat. Mech. Anal. 9, 381.

Happel, J. \& Brenner, H. 1973 Low Reynolds Number Hydrodynamics. Noordhoff.

JEFFERY, G. B. 1912 On a form of the solution of Laplace's equation suitable for problems relating to two spheres. Proc. R. Soc. A 87, 109.

JeFFeRY, G. B. 1922 The rotation of two circular cylinders in a viscous fluid. Proc. R. Soc. A 101, 169.

KaPlun, S. 1957 Low Reynolds number flow past a circular cylinder. J. Math. Mech. 6, 595.

Ladyzhenskaya, O. A. 1963 The Mathematical Theory of Viscous Incompressible Flow. Gordon \& Breach, New York.

LAmв, H. 1932 Hydrodynamics, 6th edn. Cambridge University Press.

LeE, S. H. \& LeaL, L. G. 1982 The motion of a sphere in the presence of deformable interface. II. A numerical study of the translation of a sphere normal to an interface. J. Colloid Interface Sci. 87, 81.

Moffatt, H. K. 1964 Viscous and resistive eddies near a sharp corner. J. Fluid Mech. $18,1$.

ObERBECK, A. J. 1876 Über stationäre Flüssigkeitsbewegungen mit Berücksichtigung der inner Reibung. J. reine angew. Math. 81, 62.

O'NEILL, M. E. 1964 A slow motion of viscous liquid caused by a slowly moving solid sphere. Mathematica 11, 67.

Oseen, C. W. 1927 Neuere Methoden und Ergbnisse in der Hydrodynamik. Leipzig, Akademische Verlag.

Proudman, I. \& Pearson, J. R. A. 1957 Expansions at small Reynolds numbers for the flow past a sphere and a circular cylinder. J. Fluid Mech. 2, 237.

Rallison, J. M. 1981 A numerical study of the deformation and burst of a viscous drop in general shear flows. J. Fluid Mech. 90, 465.

Robertson, C. R. \& ACrivos, A. 1970 Low Reynolds number shear flow past a rotating circular cylinder. Part 1. Momentum transfer. J. Fluid Mech. 40, 685.

Shintani, K., Umemura, A. \& Takano, A. 1983 Low Reynolds number flow past an elliptic cylinder. J. Fluid Mech. 1367, 277.

Umemura, A. 1982 Matched-asymptotic analysis of low Reynolds number flow past two equal circular cylinders. J. Fluid Mech. 121, 345.

Van Dyke, M. 1975 Perturbation Methods in Fluid Mechanics. Parabolic.

YANo, H. \& KIEDA, A. 1980 An approximate method for solving two-dimensional low Reynolds number flow past arbitrary cylindrical bodies. J. Fluid Mech. 97, 157.

Youngren, G. K. \& ACrivos, A. 1975 Stokes flow past a particle of arbitrary shape : a numerical method of solution. J. Fluid Mech. 69, 377. 